\title{
Mono- $X$ versus direct searches: simplified models for dark matter at the LHC
}

\author{
Seng Pei Liew, ${ }^{a}$ Michele Papucci, ${ }^{b, c}$ Alessandro Vichi ${ }^{d}$ and Kathryn M. Zurek ${ }^{b, c}$ \\ ${ }^{a}$ Department of Physics, University of Tokyo, \\ Bunkyo-ku, Tokyo 113-0033, Japan \\ ${ }^{b}$ Theoretical Physics Group, Lawrence Berkeley National Laboratory, \\ Berkeley, CA 94720, U.S.A. \\ ${ }^{c}$ Berkeley Center for Theoretical Physics, University of California, \\ Berkeley, CA 94720, U.S.A. \\ ${ }^{d}$ Institute of Physics, École Polytechnique Fédérale de Lausanne, \\ CH-1015, Lausanne, Switzerland \\ E-mail: liew@hep-th.phys.s.u-tokyo.ac.jp, mpapucci@lbl.gov, \\ alessandro.vichi@epfl.ch, kmzurek@lbl.gov
}

ABSTRACT: We consider simplified models for dark matter (DM) at the LHC, focused on mono-Higgs, $-Z$ or $-b$ produced in the final state. Our primary purpose is to study the LHC reach of a relatively complete set of simplified models for these final states, while comparing the reach of the mono- $X$ DM search against direct searches for the mediating particle. We find that direct searches for the mediating particle, whether in di-jets, jets $+\mathbb{E}_{T}$, multi$b+\mathbb{E}_{T}$, or di-boson $+\mathbb{E}_{T}$, are usually stronger. We draw attention to the cases that the mono- $X$ search is strongest, which include regions of parameter space in inelastic DM, two Higgs doublet, and squark mediated production models with a compressed spectrum.

KEYWORDS: Phenomenological Models

ARXIV EPRINT: 1612.00219 


\section{Contents}

1 Introduction 1

2 Simplified models for mono- $X \quad 4$

2.1 "Inelastic" dark matter $\quad 7$

2.2 Two Higgs Doublet Model 11

$\begin{array}{lll}2.3 & \text { Squarks with mono- } Z & 16\end{array}$

$\begin{array}{lll}2.4 & \text { Sbottoms with mono- } b \text {, mono- } h \text { and mono- } Z & 19\end{array}$

$\begin{array}{ll}2.5 s \text {-channel vector mediator } & 19\end{array}$

$2.6 s$-channel scalar mediator $\quad 23$

$\begin{array}{lll}2.7 & \text { Inelastic squarks } & 24\end{array}$

3 Conclusions $\quad 25$

$\begin{array}{ll}\text { A Experimental analyses and simulation details } & 28\end{array}$

$\begin{array}{ll}\text { B } 14 \mathrm{TeV} \text { projections } & 29\end{array}$

\section{Introduction}

Dark matter (DM) production at colliders is a potentially powerful complementary probe to searches for DM in direct and indirect detection experiments. Traditionally, searches for DM at colliders have focused on the signatures of DM candidates belonging to simple, non-singlet representation of the Standard Model $(\mathrm{SM})$ weak gauge group $\mathrm{SU}(2) \times \mathrm{U}(1)$, motivaved by the most popular incarnations of the weakly interative massive particle (WIMP) ideas, such as the neutralino in supersymmetry (SUSY). More recently, however, the idea that the LHC can search for WIMP DM in more general types of theories and interactions has gained traction. That one can look for DM via a jet, photon or $Z$-boson recoiling off missing energy has a long history [1-7].

Casting these bounds in the context of an effective field theory (EFT) allows one to compare the results from a collider in a straightforward way to direct and indirect detection constraints [8-14] simply by placing a bound on the scale of the EFT operator, $\Lambda$, that can be easily ported from one type of DM search experiment to the next. Perhaps because of this ease of comparison to direct and indirect detection experiments, DM searches at the Large Hadron Collider (LHC) have gained popularity, and the EFT framework has been utilized in many LHC searches at Run I.

It is clear, however, that the typical momenta exchanged in the collision processes probed at colliders such as the LHC are often beyond the values of $\Lambda$ that can be bounded, rendering a naive EFT characterization of DM searches at colliders invalid in many cases. 
Effective operators within the EFT framework are generated by integrating out heavy mediators at a scale $\Lambda$ in the UV-complete theory; a lower limit on $\Lambda$ can be derived selfconsistently if the energy scale of the processes used to constrain the theory is smaller than $\Lambda$. Further discussions and more detailed analyses of this issue can be found in [15-24]. For this reason, the collider limits obtained using the EFT approach cannot be straightforwardly used, for example, to compare with limits obtained from direct detection experiments. Various prescriptions to overcome these issues can be found, e.g., in [17, 25-28].

These statements are especially true once constraints on the mediating particle are taken into account, generally forcing one either out of the LHC reach or out of regime of validity of the EFT (e.g. [21, 24]). Identifying the regions where mono- $X$ searches provide the strongest constraint is therefore important for developing a DM LHC search program. For example, di-jet searches for the particle mediating the DM production place such strong constraints on the quark-mediator coupling that, in order for the DM-mediator coupling to be perturbative but still constrained by mono-jet searches, one finds the mediator must, in most cases, be produced on-shell. For the purpose of DM direct detection experiments, a given scattering cross-section will map to different parameter points that may have different exclusion status between mono-jet and di-jet LHC searches, thus requiring additional assumptions.

Therefore, in order to interpret DM search results at colliders adequately, simplified models should be employed [28]. Simplified models are UV-complete models that do not necessarily represent the full theory, but enable one to study the kinematics and topologies of DM production at the LHC in a precise manner. Moreover, the sensitivity comparisons between collider and direct detection limits can be performed accurately.

Simplified models immediately suggest that other signatures, apart from looking for DM recoiling against a visible SM particle, must be considered. Searching directly for the mediator of the SM-DM interaction may generally be more powerful for constraining the parameter space. For example, returning to the earlier example, assuming that the mediator is coupled to both quarks and DM, where the monojet search is expected to be important, models with $t$-channel DM production (squark mediator) are constrained by jets plus missing transverse energy $\left(\mathbb{E}_{T}\right)$ searches, while models with $s$-channel DM production ( $Z^{\prime}$ mediator) are constrained by di-jet searches. Various aspects of such simplified models have been studied extensively in the literature ${ }^{1}[18-24,31-39]$.

Simplified models for mono- $X$ searches, where here $X$ will be taken to be an object different from a jet, such as mono-Higgs [40-43], mono- $W$ [44], $-Z[6,45]$, and $-b[46,47]$ have received comparatively less attention. Understandably, one does not expect DM to be produced copiously while radiating from the initial-state a particle such as Higgs, Z or W at the LHC. In most cases, DM production with a jet from the initial state imposes the most stringent constraints. Even so, as dedicated searches for various mono- $X$ channels have already been performed [48-54] and will be extensively carried on in the current and future LHC runs, it is important and timely to consider a relatively exhaustive set of simplified models that give rise dominantly to such mono- $X$ signals. A systematic study considering

\footnotetext{
${ }^{1}$ For a comprehensive list of references, see [28-30].
} 
a broad range of simplified models is still lacking in the literature. The present work aims to bridge this gap and propose a comprehensive set of simplified models that characterizes mono- $X$ searches. In the following, we focus on the interplay of mono- $X$ limits with other collider searches as well as their phenomenological implications. We also provide UV completions of these DM production topologies. Table 1 shows diagrammatically the simplified models in consideration for mono-Higgs and mono- $Z$ as well as the models' constraints from other collider searches. In general, many models which feature a mono- $Z$ signal also have a mono- $W$ signal. For most of our analysis, we focus on singlet DM where there is only mono- $Z$ and mono- $H$ signals; the exception is the "inelastic squark" model, where the topology demands the presence of both mono- $Z$ and mono- $W$ signatures. In general, however, the constraint on the production cross-section times branching fraction is weaker for mono- $W$ as compared to mono- $Z$, rendering the former less powerful, unless the latter is strongly suppressed for, e.g., kinematic reasons. We also do not further consider mono- $\gamma$ searches $[7,55]$. When the photon is radiated from the initial state, the constraint is generically weaker than when a jet is radiated from the initial state. The other options are that that photon is radiated from the mediator or from the final state. Since the final state is charge neutral, the latter does not occur at tree level. The photon may instead be radiated from a charged non-colored mediating particle. ${ }^{2}$ In this case a charged particle must be produced in the final state as well, which must decay to additional charged SM states. These may be lost if they are sufficiently soft, but in this case, it has been shown that mono- $X$ searches alone are not very powerful [56], although they may provide stronger limits if complemented with other signatures present in the event, such as a soft lepton or a disappearing track [57]. The only exception is if the mediating particle is present in a $t$-channel in the vector-boson-fusion (VBF) topology [58]. We leave the study of the corresponding search of two forward jets and a single central photon $+\mathbb{E}_{T}$ to future work.

Among possible other mono- $X$ searches there are also those where $X$ is a bottom or top quark. Mono- $b$ searches are very effective for models where the mediator preferentially couples to the third generation, such as Higgs-like particles. The correspondence between mono- $b$ and direct searches for this type of $s$-channel model has been thoroughly investigated in [47]. In this work, we will consider a simplified model with $t$-channel mediator (sbottom), which, as will be shown below, also plays a role in mono- $h$ and mono- $Z$ searches. Table 2 shows diagrammatically the mono- $b$ topology as well as the relevant direct searches considered in this work. In the case of mono- $t$ searches the only simplified models producing sizable signals at tree level are divided in two categories depending on whether mono- $t$ is resonantly produced, as in $R$-parity violating (RPV) SUSY, or non-resonantly produced via a $t$-channel top quark [59-63]. Strictly speaking, the RPV SUSY scenario does not have a dark matter candidate, as the lightest neutralino is not stable on cosmological time scales. Moreover, both scenarios involve flavor-changing neutral interactions, which potentially lead to stringent flavor constraints. Furthermore, key direct searches for the RPV case involve displaced stop decays and apart from a few (very powerful) searches performed

\footnotetext{
${ }^{2}$ If the mediating particle is also colored, mono-jet searches tend to provide stronger limits than the corresponding mono-photon ones.
} 
at Run I, both experiments are ramping up search efforts for long-lived particles in Run II. Given these complications, we leave the detailed study of mono- $t$ signatures elsewhere.

In table 3, we summarize our main results: for each mono- $X$ search studied in this paper we list the simplified model where it has reach. We omit simplified models where a given search can only exclude parameter space already ruled out by a different analysis.

The $s$-channel $Z^{\prime}$ and Higgs mediated models are briefly commented on in the next section without performing further mono- $X$ analysis as they have been studied in detail previously $[41,42]$. Our primary purpose there is to compare the mono- $X$ analysis against other ways to look for the mediator and/or the DM particle at the LHC. In each of the subsequent models, we compare the strength of mono- $Z$ and mono-Higgs against each other and the constraints from other searches, such as di-jet, jets $+\mathbb{E}_{T}$, mono-jet, di-boson $+\mathbb{E}_{T}$ and mono- $b$, whenever they are relevant. These results will serve as a guideline to both theorists and experimentalists for optimizing mono- $X$ searches. For reference, we list all relevant collider searches utilized in our analysis in table 4.

For illustrating our results, we focus here on Run I searches, since a complete set of both mono- $X$ and direct searches performed with similar amounts of integrated luminosity has been performed. At the time of writing this is not yet the case for Run II analyses with approximately $13 \mathrm{fb}^{-1}$. We checked and found the set of analyses released with 2015 data do not significantly increase the Run I limits. Therefore in the following, we will perform comparisons among different searches with $8 \mathrm{TeV}$ data and use the available $13 \mathrm{TeV}$ searches to validate the procedure we use to make our projections for the future reach, at $300 \mathrm{fb}^{-1}$, as described in appendix B. ${ }^{3}$ The study presented here can nevertheless be updated with new Run II analyses once those are completely available.

The outline of this paper is as follows. In the next section we summarize the models and analyses utilized in our comparison of mono- $X$ searches against various searches for the mediating particle. In the following subsections, we then systematically compare the constraints for each model in table 1 and 2 from mono- $X$ to various searches for resonances, as well as for supersymmetry. Our goal is to highlight the classes of models where mono- $X$ constraints shed the most new light on new physics, beyond what is already constrained by more standard types of searches. Finally, we conclude.

\section{Simplified models for mono- $X$}

Before describing the details of each simplified model, we discuss the general properties and assumptions made on the models considered here. We require that:

- the DM is a fermionic singlet under the SM gauge group;

- the mono- $X$ signatures are produced by tree-level topologies,

- the model have the smallest number of mediating particles for each mono- $X$ topology we consider.

\footnotetext{
${ }^{3}$ The only exception to this rule is a boosted di-jet analysis performed for the first time in Run II with $2.7 \mathrm{fb}^{-1}$. This analysis is important for improving the low mass limits, and we utilize it because with this luminosity we expect similar constraints as with $20 \mathrm{fb}^{-1}$ at $8 \mathrm{TeV}$.
} 


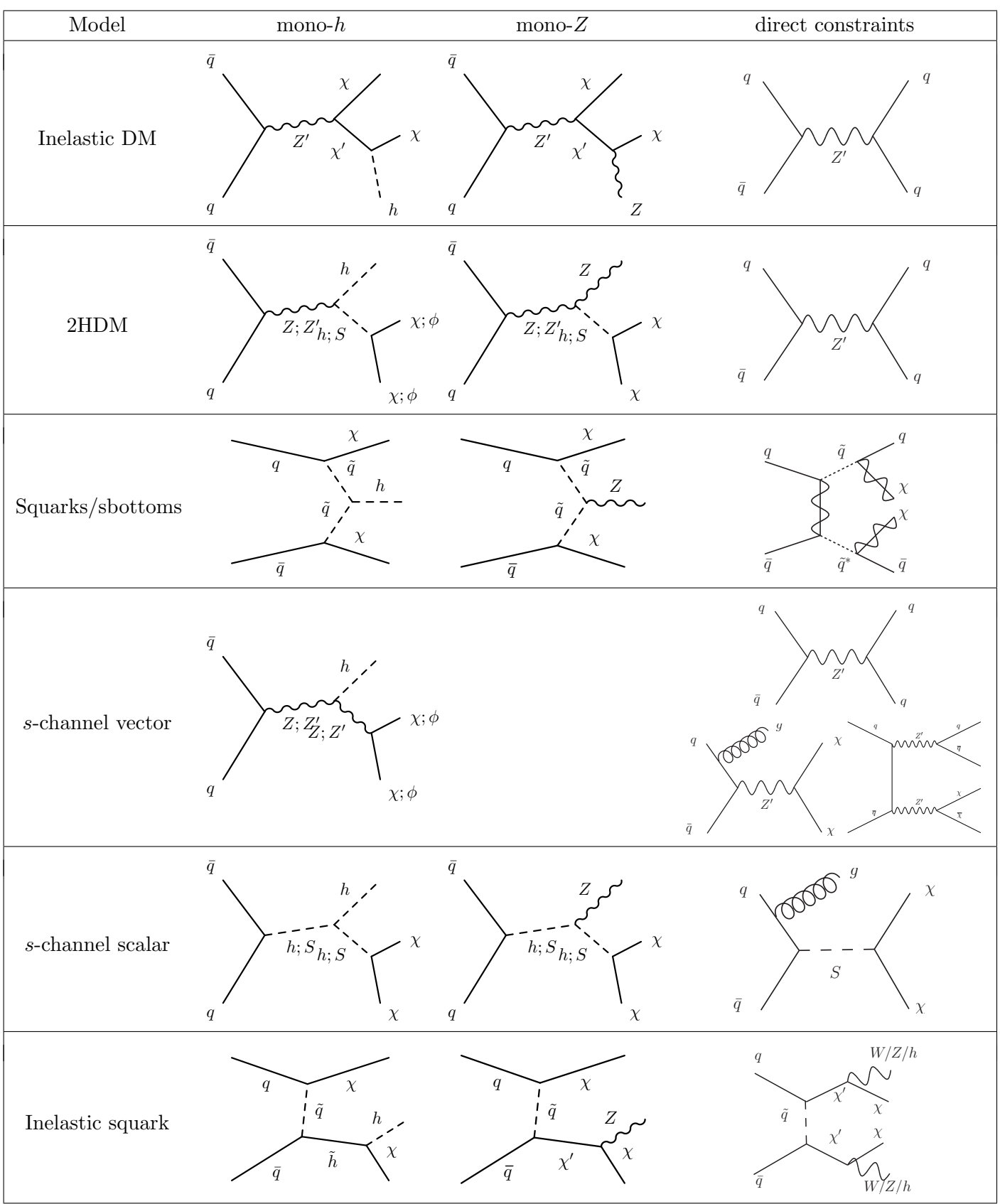

Table 1. Summary of mono-Higgs and mono- $Z$ topologies, as well as the corresponding relevant direct searches considered in this work.

We only consider pair production of DM at colliders given that DM is stable on timescales the order of the lifetime of the Universe. An $s$-channel vector (scalar) mediator is denoted as $Z^{\prime}(S)$. We also use the notation of SUSY whenever a SUSY analogue is applicable to our simplified models. For example, $\tilde{q}$ denotes the $t$-channel colored mediator that couples to a quark $(q)$ and DM $(\chi)$. Other auxiliary particles may be needed for constructing our simplified models. They are defined accordingly in the respective subsection describing the details of the simplified model. 


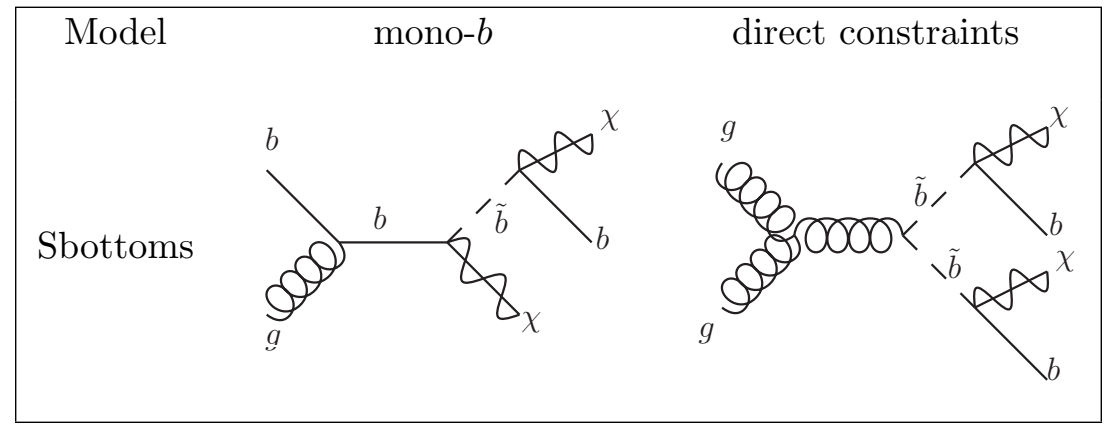

Table 2. Summary of mono- $b$ topology, as well as the corresponding relevant direct search considered in this work.

\begin{tabular}{|c|c|}
\hline Search & Model where it matters \\
\hline mono- $h$ & Inelastic DM, 2HDM \\
\hline mono- $z$ & Inelastic DM, 2HDM \\
\hline mono-jet & Squark mediated production, compressed spectrum \\
\hline mono- $b$ & Sbottom mediated production, compressed spectrum \\
\hline
\end{tabular}

Table 3. Summary of results: for each mono- $X$ search we list the models where the analysis can exclude part of the parameter space not already ruled out by some other search.

\begin{tabular}{|c|c|c|}
\hline Simplified model & searches compared & method \\
\hline Inelastic DM & mono- $h$ & full recasting \\
& mono- $z$ & full recasting \\
\hline 2 HDM & mono- $h$ & full recasting \\
& mono- $z$ & full recasting \\
\hline Squarks & mono- $z$ & full recasting \\
$\left(u_{L, R}, d_{L, R}, c_{L, R}, s_{L, R}\right)$ & mono-jet & results of [24] \\
& multi-jet $+\mathbb{E}_{T}$ & results of [24] \\
\hline Sbottom & mono- $z$ & full recasting \\
& mono- $b$ & simplified model [64] \\
& multi- $b$ jets $+\mathbb{E}_{T}$ & simplified model [65] \\
\hline$s$-channel & mono- $h$ & results of [41] \\
scalar mediator & mono-jet & full recasting \\
\hline$s$-channel & mono- $h$ & results of [41] \\
vector mediator & mono-jet & full recasting \\
& multi-jet $+\mathbb{E}_{T}$ & full recasting \\
\hline Inelastic Squarks & mono- $h$ & full recasting \\
& mono- $z$ & full recasting \\
& diboson $+\mathbb{E}_{T}$ & simplified model [66, 67] \\
& bosons + jets $+\mathbb{E}_{T}$ & simplified model [68] \\
\hline
\end{tabular}

Table 4. Summary of simplified models and analyses considered in this work. The last column indicates whether we perform a full reinterpretation, use the results published by the experimental collaborations, or utilize previous work in the literature. 
Given this set of rules, one can find the list of all the possible topologies and embed each of them in the minimal incarnation of a simplified model as defined above. We relax the requirement of singlet DM only for the case of the inelastic squark model, where the topology we consider requires the DM to take on SM quantum numbers. These requirements are also easy to understand: focusing on singlet DM stems from the fact that searches for DM belonging to weak doublets or triplets are more mature due to the extensive program for SUSY searches. Restricting our focus to tree level topologies and keeping the number of mediators to a minimum instead originates from the attempt to maximize the reach potential of mono- $X$ searches in comparison to direct searches for the mediators.

For the purpose of illustrating the strength of mono- $X$ and direct searches relevant to these simplified models, we either perform Monte Carlo event simulation, or make use of results of previous works in the literature and as presented by the experimental collaborations. We do not perform full scans in the parameter space of each model, but rather focus on slices of parameter space we believe are highlighting the main qualitative features of the comparisons between mono- $X$ and other searches. A full parameter scan can in principle be performed but it is beyond the scope of this work. We summarize the methods and analyses employed for the simplified models in table 4. The details of the experimental analyses and our simulations, as well as our method of obtaining $14 \mathrm{TeV}$ projections, are elaborated in appendices A, B.

\section{1 "Inelastic" dark matter}

We begin by considering a Higgs or $Z$ radiated in the final state through the process $\chi^{\prime} \rightarrow \chi h$ or $\chi^{\prime} \rightarrow \chi Z$, where $\chi^{\prime}$ and $\chi$ are produced via a resonant $Z^{\prime}$. Here, $\chi^{\prime}$ is an "excited" state of DM $\chi$ that decays to DM along with a Higgs or $Z$. These processes arise from interaction Lagrangians of the form $Z_{\mu}^{\prime} \chi^{\prime} \gamma^{\mu} \chi, Z_{\mu} \chi^{\prime} \gamma^{\mu} \chi$ and $h \chi \chi^{\prime}$. In order for mono- $h$ or mono- $Z$ to be dominant, production of $\chi^{\prime} \chi^{\prime}$ (which leads to di-boson signatures) and $\chi \chi$ (which will be dominated by mono-jet) must be suppressed relative to $\chi \chi^{\prime}$. We discuss a concrete model where the mono-boson signature dominates.

For concreteness, we focus on the case where only the right-handed up-quarks (all three generations) are charged under a new gauge symmetry. ${ }^{4}$ In addition, a new SM singlet Dirac fermion $\psi$ charged under $\mathrm{U}(1)_{Z^{\prime}}$ is introduced as a doublet of DM. Moreover, we introduce a SM singlet scalar $S$ that is charged under $\mathrm{U}(1)_{Z^{\prime}}$. It plays the roles of giving the $Z^{\prime}$ a mass and acting as a "portal" to the Higgs. We also assume that some of the SM quarks are charged under it, to allow a $q \bar{q} Z^{\prime}$ coupling. In the following we will allow only the right-handed up quark to carry $\mathrm{U}(1)_{Z^{\prime}}$ charge which we fix to $1 / 2$. We take the DM $(\psi=(\eta \bar{\xi}))$ Lagrangian to be:

$$
\mathcal{L}_{\mathrm{DM}}=\bar{\psi}\left(i \not D-m_{\psi}\right) \psi-\left(\lambda_{1} S^{*} \eta \eta+\lambda_{2} S \xi \xi+\text { h.c. }\right),
$$

\footnotetext{
${ }^{4}$ This model requires the introduction of extra (spectator) fermions to achieve anomaly cancellation. The upper limit of the masses the spectator fermions are $M_{\text {spectator }}<\left(64 \pi^{2} / g_{q q Z^{\prime}}^{3}\right) M_{Z^{\prime}}$, where $g_{q q Z^{\prime}}$ is the coupling between SM quarks and the new gauge boson $Z^{\prime}$, and $M_{Z^{\prime}}$ is the mass of $Z^{\prime}$ [31, 69]. To focus on the more generic collider signatures of the model, we consider these spectator fermions to be sufficiently heavy (achievable by saturating the aforementioned mass upper limit), such that LHC constraints on them are avoided.
} 
where $D_{\mu}=\partial_{\mu}+i g_{X} q_{\psi} \hat{X}_{\mu}$. We define new bases $\chi_{1}, \chi_{2}=1 / \sqrt{2}(\eta \mp \xi)$ and new couplings $\lambda_{ \pm}=\lambda_{1} \pm \lambda_{2}$ such that, after the $\mathrm{U}(1)_{Z^{\prime}}$ symmetry is spontaneously broken, the fermion bilinear terms are written as:

$$
\mathcal{L}_{\mathrm{DM}}^{\mathrm{bi}}=-\frac{1}{2}\left(-m_{\psi} \chi_{1}^{2}+m_{\psi} \chi_{2}^{2}+\lambda_{+}\langle S\rangle \chi_{1}^{2}+\lambda_{+}\langle S\rangle \chi_{2}^{2}\right)-\lambda_{-}\langle S\rangle \chi_{1} \chi_{2}+\text { h.c.. }
$$

Introducing the mass eigenstates (with abbreviations $c_{\chi} \equiv \cos \theta_{\chi}, s_{\chi} \equiv \sin \theta_{\chi}$ ),

$$
\left(\begin{array}{l}
\chi_{1} \\
\chi_{2}
\end{array}\right)=\left(\begin{array}{cc}
c_{\chi} & s_{\chi} \\
-s_{\chi} & c_{\chi}
\end{array}\right)\left(\begin{array}{c}
\chi \\
\chi^{\prime}
\end{array}\right)
$$

the mixing angle and mass eigenvalues are given by

$$
\begin{aligned}
\tan 2 \theta_{\chi} & =\frac{\lambda_{-}\langle S\rangle}{m_{\psi}}, \\
M_{\chi, \chi^{\prime}}^{2} & =\lambda_{+}\langle S\rangle \mp \sqrt{m_{\psi}^{2}+\lambda_{-}^{2}\langle S\rangle^{2}} .
\end{aligned}
$$

In the new basis, $\chi$ is the DM candidate while $\chi^{\prime}$ is the "excited" state of DM.

$S$ and $Z^{\prime}$ mix with the SM Higgs and $Z$ respectively, and facilitate the mono- $X$ processes $\chi^{\prime} \rightarrow \chi h$ and $\chi^{\prime} \rightarrow \chi Z$. The interaction of the scalar field $\delta S \equiv \sqrt{2}(S-\langle S\rangle)$ with the DM doublet is as follows:

$$
\mathcal{L}_{\mathrm{DM}}^{\mathrm{sc} . i n t}=\frac{\lambda_{+}}{\sqrt{2}} \delta S\left(\chi^{\prime 2}+\chi^{2}\right)+\frac{\lambda_{-}}{\sqrt{2}}\left(c_{\chi}^{2}-s_{\chi}^{2}\right) \delta S \chi \chi^{\prime}+\text { h.c. },
$$

while the interaction of $Z^{\prime}$ with the DM doublet is:

$$
\bar{\eta} \not{X} \eta-\bar{\xi} \not{X} \xi=2 g_{X} q_{\psi} s_{\chi} c_{\chi}\left(\bar{\chi}^{\prime} \not{X} \chi^{\prime}-\bar{\chi} \not{X} \chi\right)-g_{X} q_{\psi}\left(c_{\chi}^{2}-s_{\chi}^{2}\right)\left(\bar{\chi} \not{X} \chi^{\prime}+\bar{\chi}^{\prime} \not{X} \chi\right) .
$$

Let us note that at the limit where the mixing angle $\theta_{\chi} \rightarrow 0$, the couplings of $Z^{\prime}$ to $\chi \chi$ and $\chi^{\prime} \chi^{\prime}$ (leading to di-boson signature) vanish, and the $\chi \chi^{\prime}$ production (leading to mono-boson signature) becomes dominant.

The $Z^{\prime}-Z$ mixing originates from the radiative corrections that lead to kinetic mixing between the $\mathrm{U}(1)$ gauge bosons:

$$
\mathcal{L}_{V}^{\mathrm{KE}}=-\frac{1}{4} \hat{X}_{\mu \nu} \hat{X}^{\mu \nu}+\frac{\epsilon}{2} \hat{X}_{\mu \nu} \hat{B}^{\mu \nu}
$$

where $\epsilon$ is expected to have the size $\epsilon \sim g_{X} g^{\prime} / 16 \pi^{2} \lesssim 10^{-3}$ from fermion loops. The Higgs sector Lagrangian of the model is written as:

$$
\begin{aligned}
\mathcal{L}_{H}= & \left|D_{\mu} H_{\mathrm{SM}}\right|^{2}+\left|D_{\mu} S\right|^{2}+m_{S}^{2}|S|^{2}+m_{H}^{2}\left|H_{\mathrm{SM}}\right|^{2} \\
& -\lambda\left|H_{\mathrm{SM}}\right|^{4}-\rho|S|^{4}-\kappa\left|H_{\mathrm{SM}}\right|^{2}|S|^{2} .
\end{aligned}
$$

$\mathrm{U}(1)_{X}$ is broken spontaneously by $\langle S\rangle$, and electroweak symmetry is broken spontaneously as usual by $\left\langle H_{\mathrm{SM}}\right\rangle=(0, v / \sqrt{2})$. The two physical Higgs bosons $h$ and $S$ mix with each other after spontaneous symmetry breaking. Whether $\chi^{\prime}$ decays to $h$ or $Z$ mainly depends 


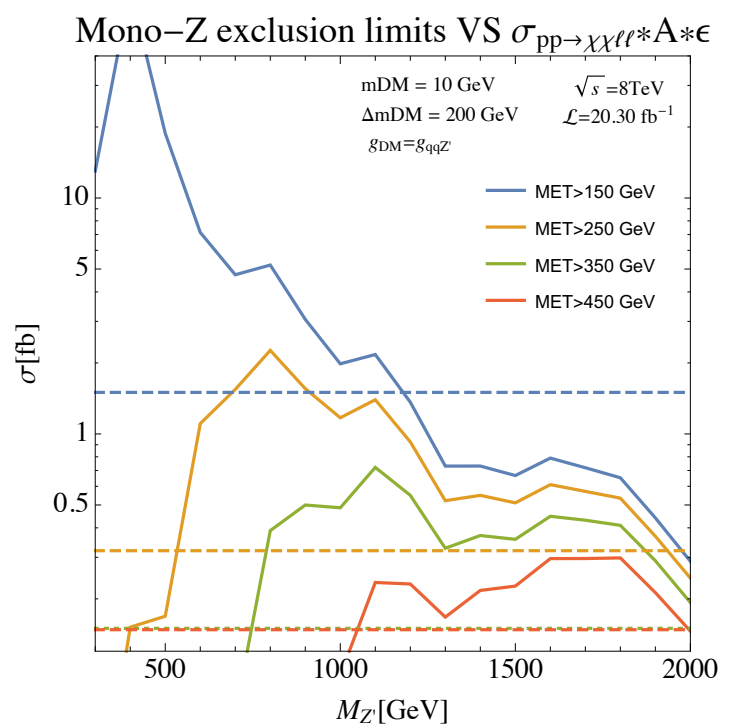

(a)

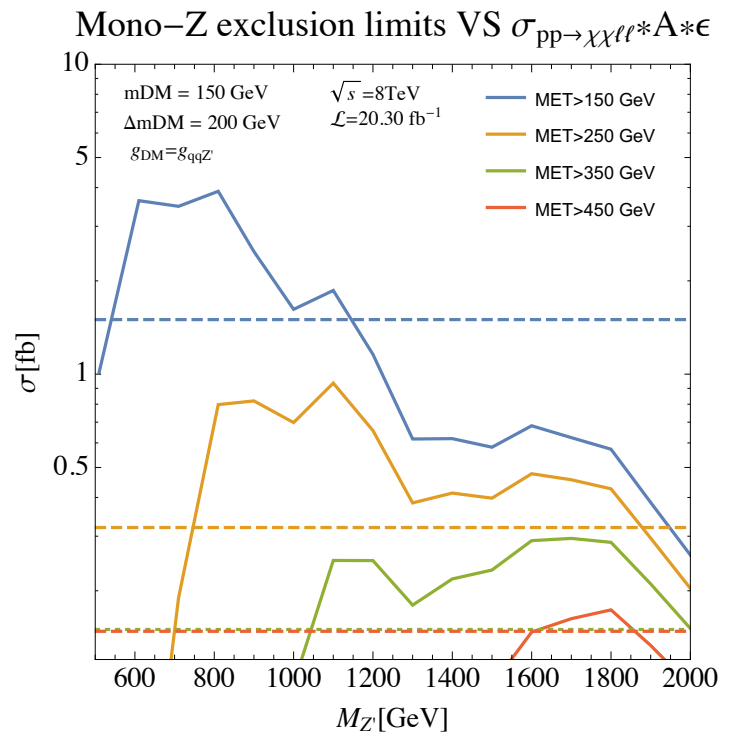

(c)

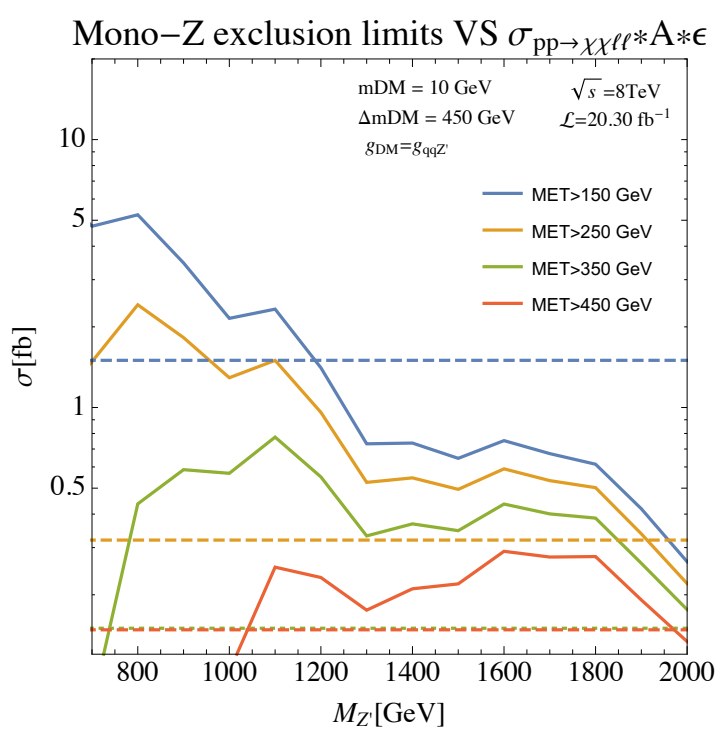

(b)

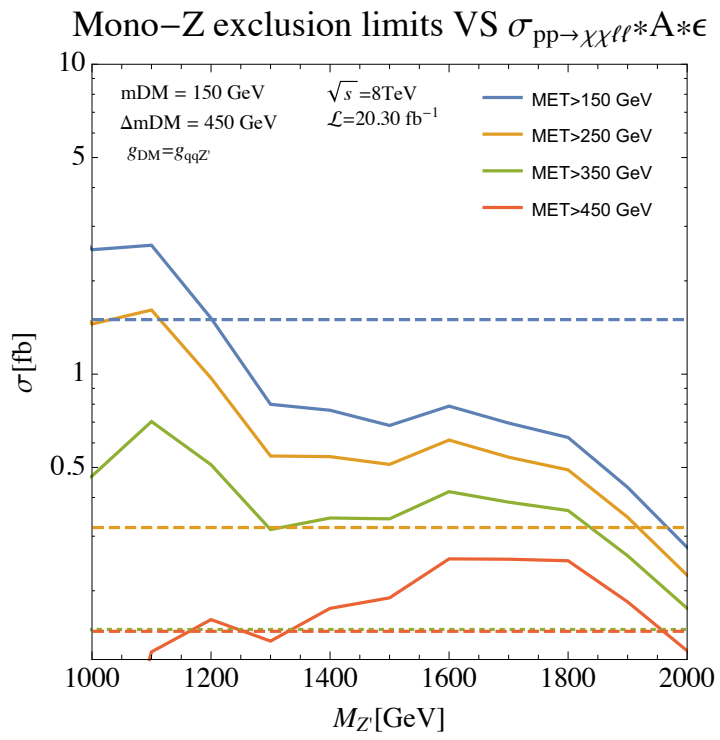

(d)

Figure 1. Inelastic DM model: mono- $Z$ exclusion cross-section at 95\% C.L., shown as dashed lines, from $8 \mathrm{TeV}$ data. $\chi^{\prime}$ is assumed to have a $100 \%$ decay to $Z \chi$. The solid lines correspond to the prediction of the model when the coupling of $Z^{\prime}$ to the quarks $g_{q q Z^{\prime}}$ is chosen to be equal to the upper limit consistent with di-jet constraints at a given $Z^{\prime}$ mass (see figure 17). Panels (a)-(d) correspond to the choice of the mass parameters $\left(m_{\mathrm{DM}}, \Delta m_{\mathrm{DM}}\right)=(10,200),(10,450),(150,200),(150,450)$ in $\mathrm{GeV}$, respectively, where $\Delta m_{\mathrm{DM}}$ is the $\chi^{\prime} \chi$ mass splitting. The four colors represent the four different $\mathbb{E}_{T}$ choices in the mono- $Z$ analysis $(150,250,350$ and $450 \mathrm{GeV})$. 


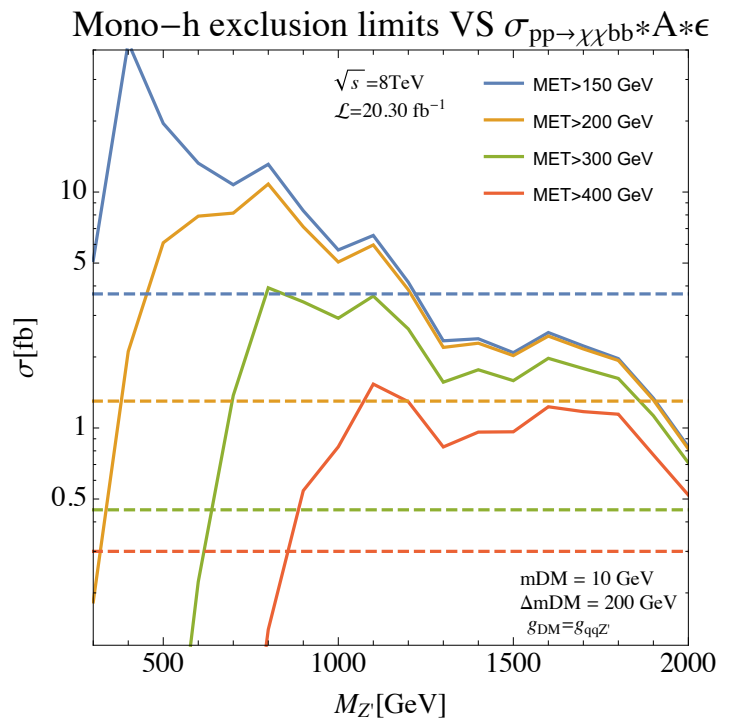

(a)

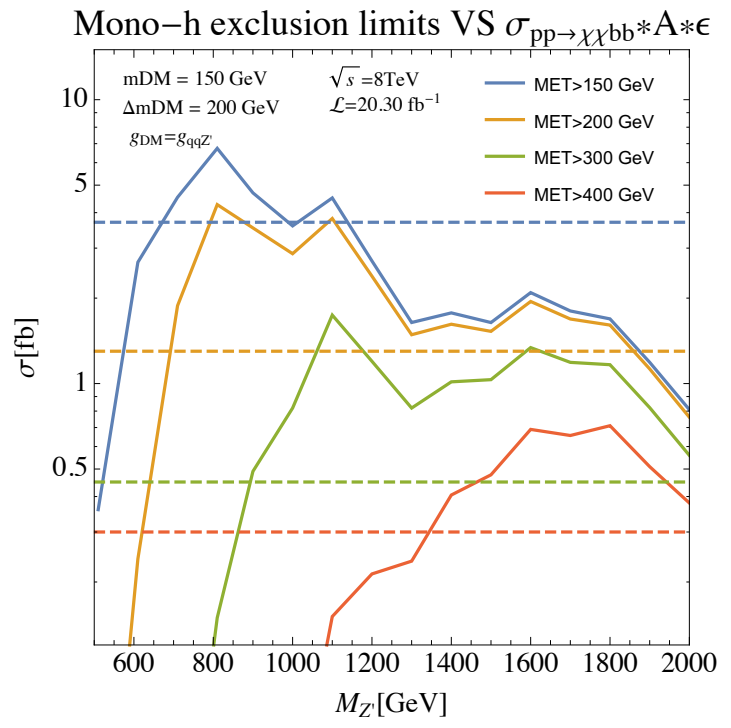

(c)

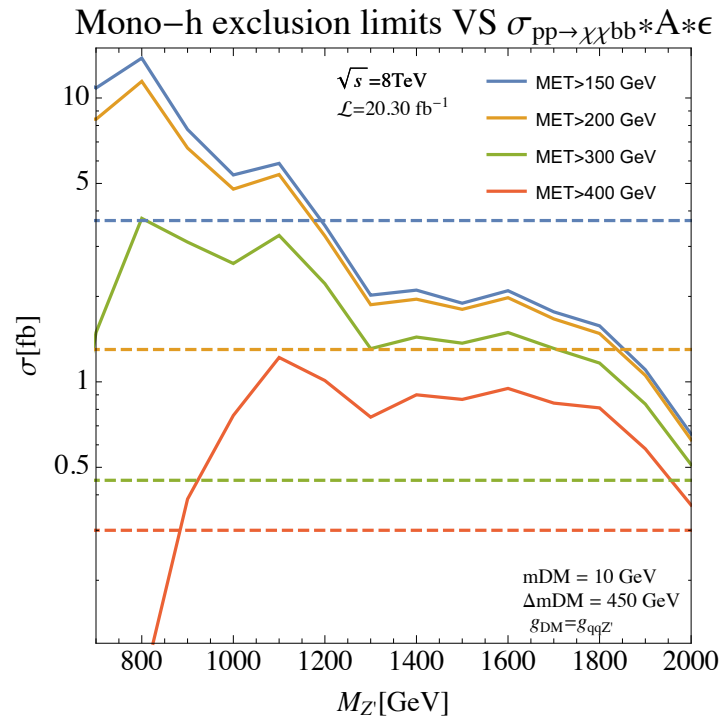

(b)

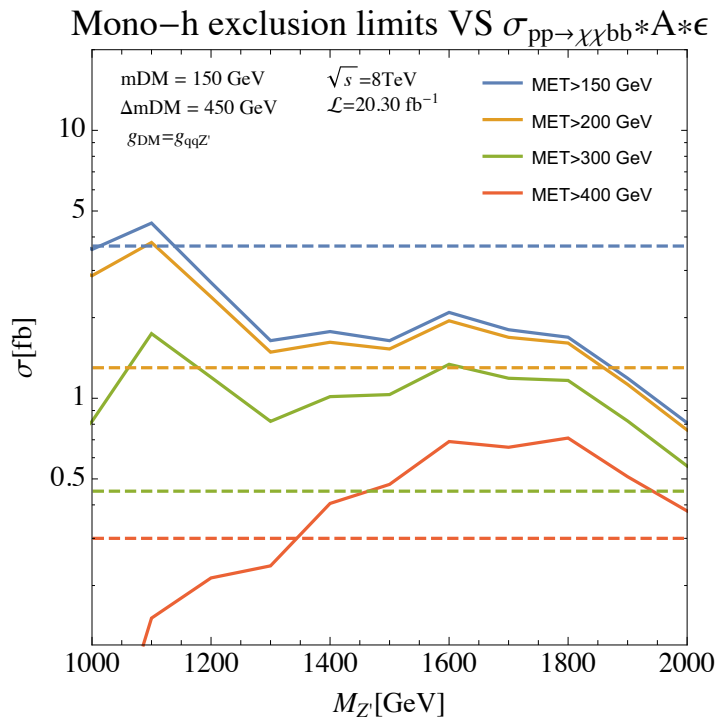

(d)

Figure 2. Inelastic DM model: mono- $h$ exclusion cross-section at 95\% C.L., shown as dashed lines, from $8 \mathrm{TeV}$ data. $\chi^{\prime}$ is assumed to have a $100 \%$ decay to $h \chi$. The solid lines correspond to the prediction of the model when the coupling of $Z^{\prime}$ to the quarks $g_{q q} Z^{\prime}$ is chosen to be equal to the upper limit consistent with di-jet constraints at a given $Z^{\prime}$ mass (see figure 17). Panels (a)-(d) correspond to the choice of the mass parameters $\left(m_{\mathrm{DM}}, \Delta m_{\mathrm{DM}}\right)=(10,200),(10,450),(150,200),(150,450)$ in $\mathrm{GeV}$, respectively, where $\Delta m_{\mathrm{DM}}$ is the $\chi^{\prime} \chi$ mass splitting. The four colors represent the four different $\mathbb{E}_{T}$ choices in the mono- $h$ analysis $(150,200,300$ and $400 \mathrm{GeV})$. 
on the value of $\epsilon$ and $\kappa$, which are in principle free parameters. We also note that the elastic scattering of DM off nucleons via $Z^{\prime}$ is suppressed as long as $\theta_{\chi}$ is small.

We compare the constraints from mono- $h$ and mono- $Z$ analyses on the cross-section times branching fraction in figures 1,2 . We investigate four benchmark points which have different combinations of DM mass $m_{\mathrm{DM}}(10 \mathrm{GeV}$ and $150 \mathrm{GeV})$ and $\Delta m_{\mathrm{DM}} \equiv m_{\chi^{\prime}}-$ $m_{\chi}(200 \mathrm{GeV}$ and $450 \mathrm{GeV})$. We can see in these two figures that both final states can be constraining, though the mono- $Z$ search with $250 \mathrm{GeV} \mathbb{E}_{T}$ cut (mono- $h$ search with $300 \mathrm{GeV} \mathbb{E}_{T}$ cut) is typically strongest. In all of the figures, we have chosen the coupling to quarks $g_{q q Z^{\prime}}$ to saturate the di-jet resonance search constraints at a given $Z^{\prime}$ mass (see figure 17$)$. In addition, we vary the DM- $Z^{\prime}$ coupling $\left(g_{\mathrm{DM}}\right)$ and show in figure 3 the $95 \%$ C.L. upper limit on the ratio $g_{\mathrm{DM}} / g_{q q Z^{\prime}}$ from the mono- $Z$ and mono- $h$ searches. We further compare future projections for $14 \mathrm{TeV}$ mono- $h$ and mono- $Z$ analyses in figure 4 , taking a $\mathbb{E}_{T}$ cut of $400 \mathrm{GeV}$, as described in appendix B. It is observed that the bounds on the production cross-section for large mediator mass is vastly improved at increased center of mass energy.

\subsection{Two Higgs Doublet Model}

We consider the resonant production of a new heavy gauge boson $Z^{\prime}$ which decays to Higgs $(Z)$ and a CP-odd (CP-even) scalar $A^{0}(H)$, as considered in [42]. The CP-odd (CP-even) scalar then is taken to exclusively decay into a pair of DM particles. The dominant mono$X$ signal is therefore mono-Higgs or mono- $Z$. In general, the simplified model Lagrangian of this topology can be written as:

$$
\begin{aligned}
\mathcal{L} \supset & g_{q} Z_{\mu}^{\prime} \sum_{i=1,2}\left(\bar{Q}_{L}^{i} \gamma^{\mu} Q_{L}^{i}+\bar{u}_{R}^{i} \gamma^{\mu} u_{R}^{i}+\bar{d}_{R}^{i} \gamma^{\mu} d_{R}^{i}\right) \\
& +\frac{1}{2} m_{Z^{\prime}}^{2} Z_{\mu}^{\prime} Z^{\prime \mu}+i \mu_{A} \partial_{\mu} A^{0} Z^{\prime \mu} h+\mu_{H} Z_{\mu}^{\prime} Z^{\mu} H .
\end{aligned}
$$

Let us consider a UV completion of this DM production topology in order to make concrete comparisons of collider constraints as well as precision electroweak constraints. Our model and analysis follow ref. [42] closely, though here we perform the mono- $Z$ analysis for the first time and update the mono- $h$ constraints with newer di-jet limits. We introduce a two Higgs doublet model $(2 \mathrm{HDM})$ with Type-II Yukawa structure $\left(H_{u}, H_{d}\right)$, i.e. $H_{u}$ couples with $u$-type quarks while $H_{d}$ couples with $d$-type quarks and charged leptons. Following ref. [42], we assume that only $H_{u}$ and $u_{R}$ are charged under the new gauge symmetry $\mathrm{U}(1)_{Z^{\prime}}$ (the charge for both $H_{u}$ and $u_{R}$ is assumed to be $1 / 2$ ). The $\mathrm{U}(1)_{Z^{\prime}}$ gauge symmetry is assumed to be broken spontaneously above the electroweak scale due to a new SM singlet scalar.

The physical Higgs bosons can be parametrized as follows:

$$
\begin{aligned}
H_{u} & =\frac{1}{\sqrt{2}}\left(\begin{array}{c}
-\sin \beta H^{+} \\
v_{u}+\cos \alpha h+\sin \alpha H+i \cos \beta A^{0}
\end{array}\right) \\
H_{d} & =\frac{1}{\sqrt{2}}\left(\begin{array}{c}
\cos \beta H^{+} \\
v_{u}-\sin \alpha h+\cos \alpha H-i \sin \beta A^{0}
\end{array}\right) .
\end{aligned}
$$


Mono-Z/h exclusion limits VS $g_{\mathrm{DM}} / g_{\mathrm{qqZ}}$

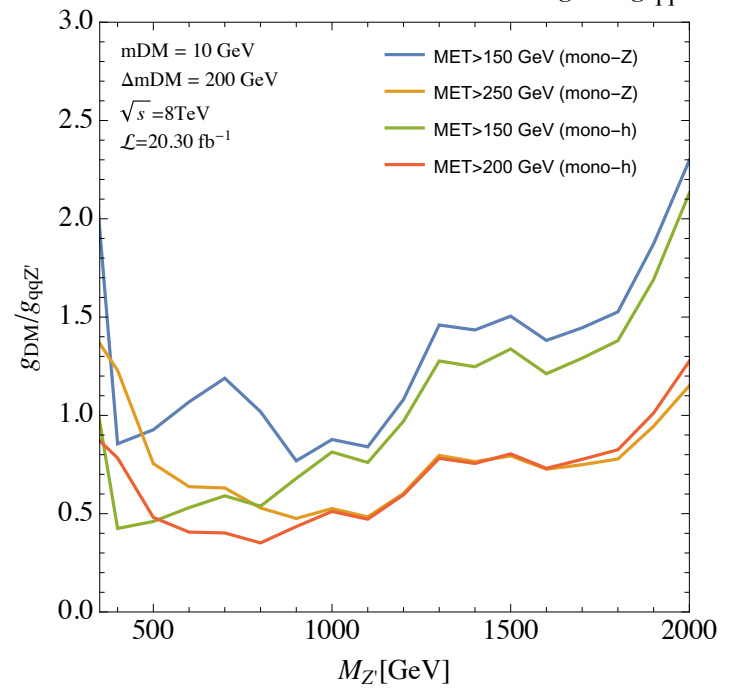

(a)

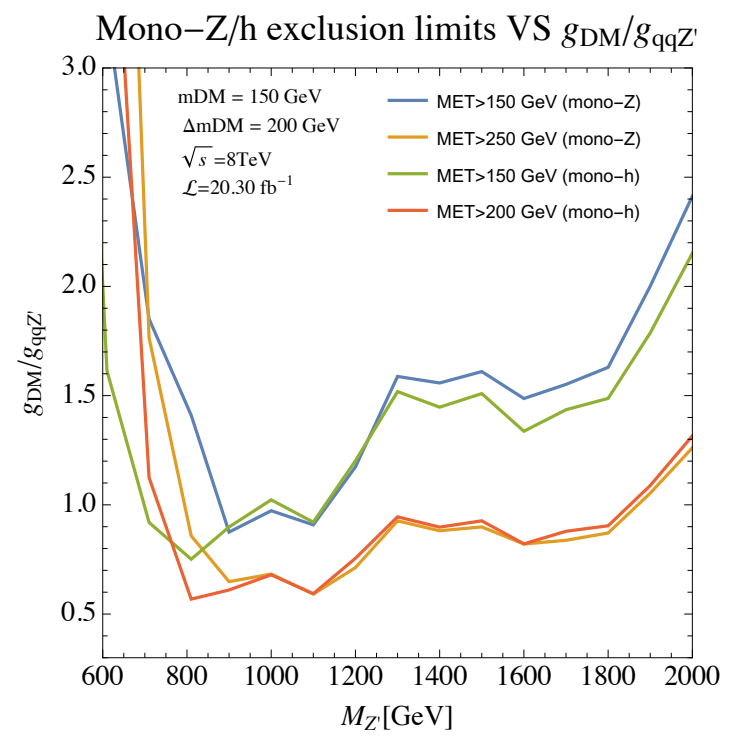

(c)

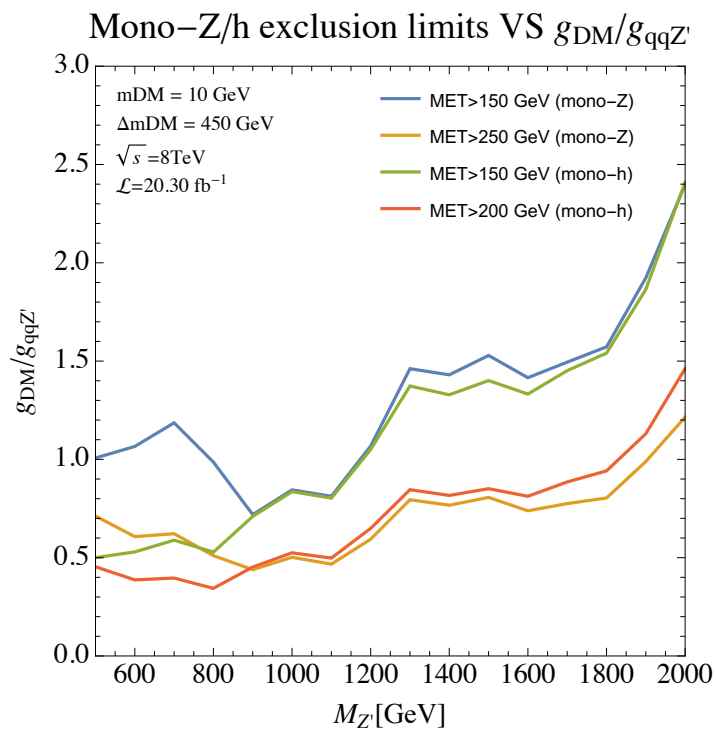

(b)

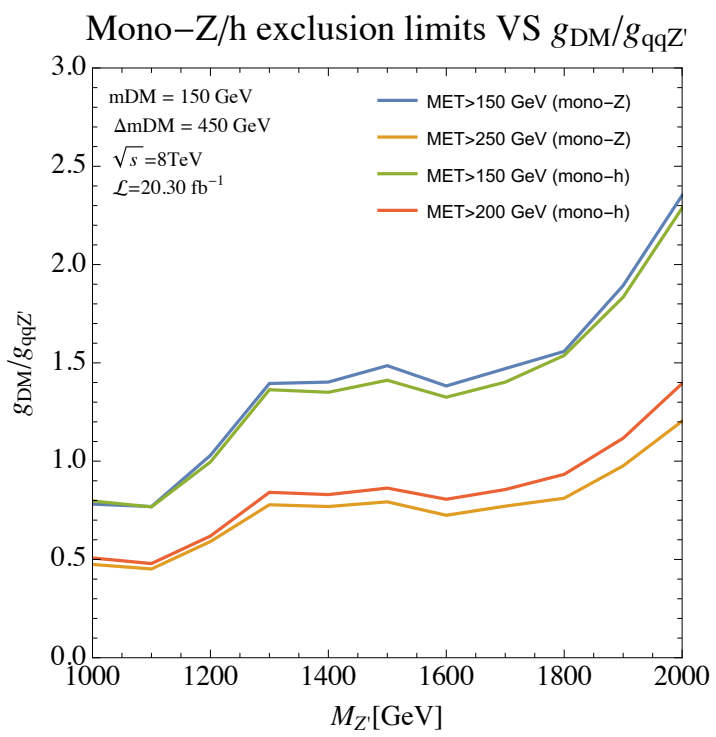

(d)

Figure 3. Inelastic DM model: constraints on the ratio of the couplings $\left(g_{\mathrm{DM}} / g_{q q Z^{\prime}}\right)$ as a function of the $Z^{\prime}$ mass from mono- $Z$ and mono- $h$ searches at $8 \mathrm{TeV}$. The coupling of $Z^{\prime}$ to the quarks $g_{q q Z^{\prime}}$ is chosen to be equal to the upper limit consistent with di-jet constraints at a given $Z^{\prime}$ mass (see figure 17). Panels (a)-(d) correspond to the choice of the mass parameters $\left(m_{\mathrm{DM}}, \Delta m_{\mathrm{DM}}\right)=$ $(10,200),(10,450),(150,200),(150,450)$ in $\mathrm{GeV}$, respectively. 


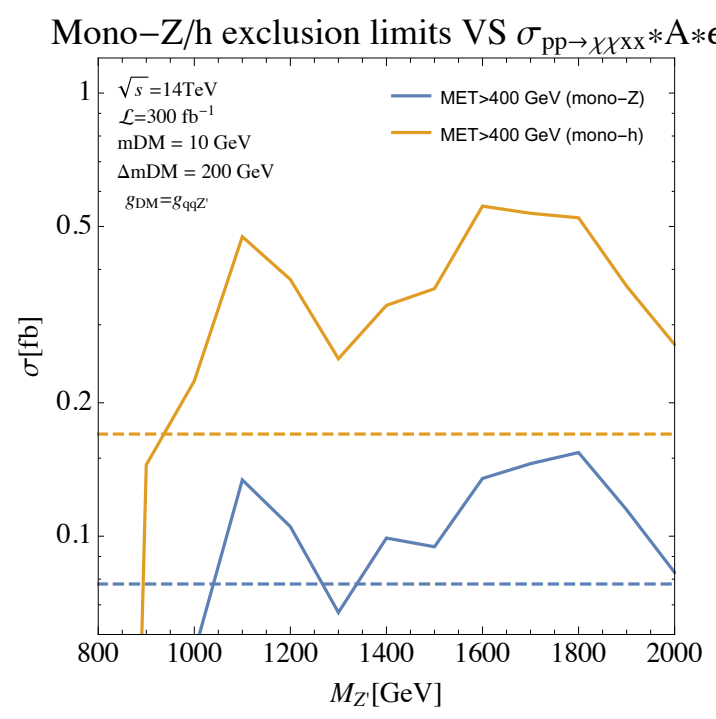

(a)

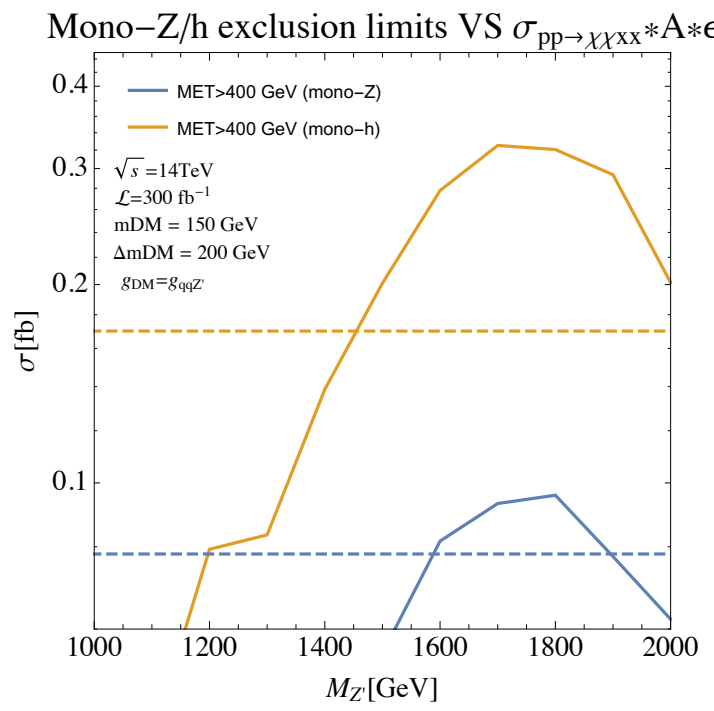

(c)

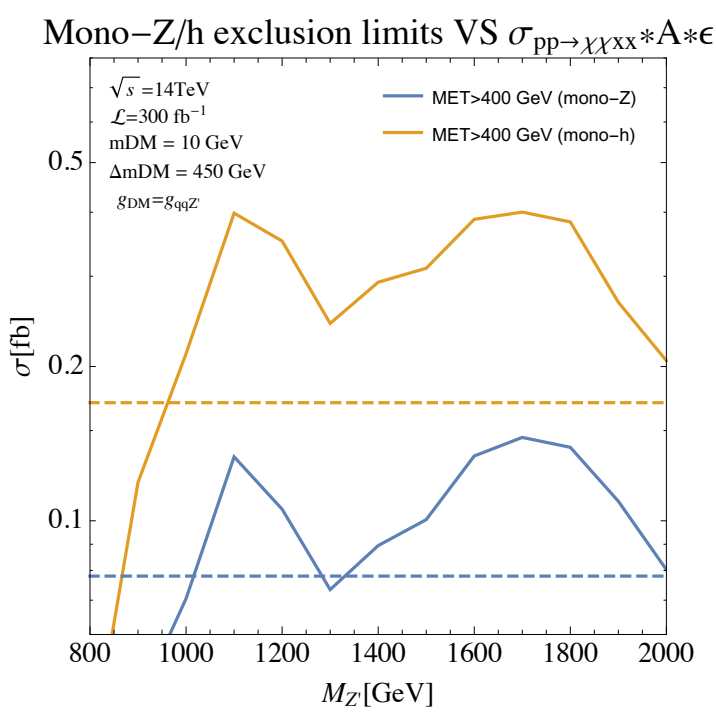

(b)

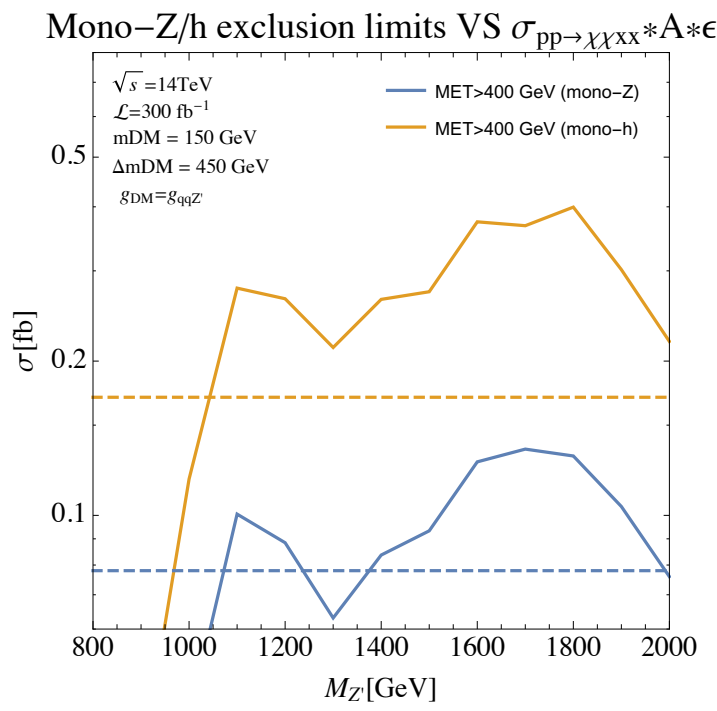

(d)

Figure 4. Inelastic DM model: 95\% C.L. mono- $Z$ and mono- $h$ exclusion cross-section (dashed lines), projected at $14 \mathrm{TeV}$ with a total integrated luminosity of $300 \mathrm{fb}^{-1}$, assuming $\mathbb{E}_{T}>400 \mathrm{GeV}$. The predictions of the inelastic DM model, when the coupling of the $Z^{\prime}$ to the quarks $g_{q q Z^{\prime}}$ are chosen to be equal to the upper limit consistent with di-jet constraints at a given $Z^{\prime}$ mass (see figure 17), is shown as solid lines. Panels (a)-(d) correspond to the choice of the mass parameters $\left(m_{\mathrm{DM}}, \Delta m_{\mathrm{DM}}\right)=(10,200),(10,450),(150,200),(150,450)$ in $\mathrm{GeV}$ respectively. 


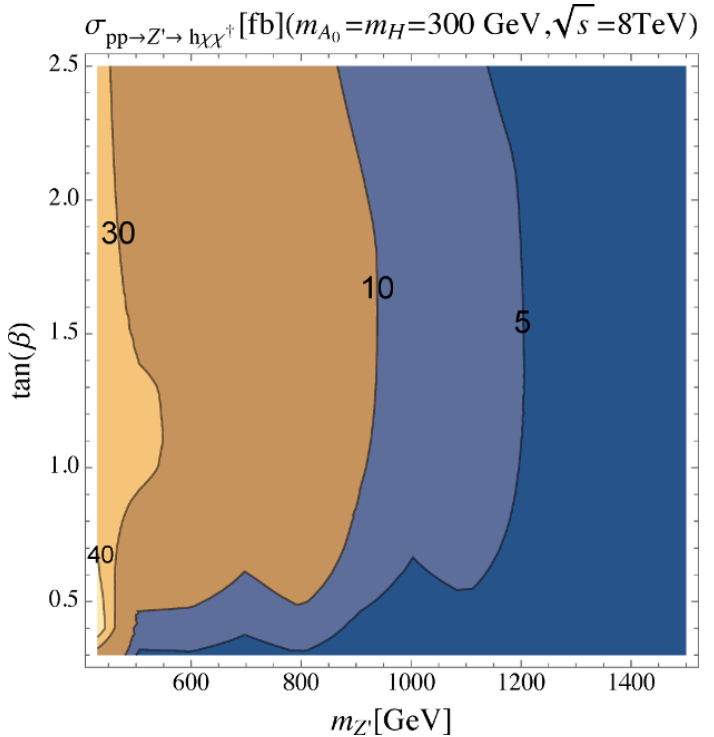

(a)

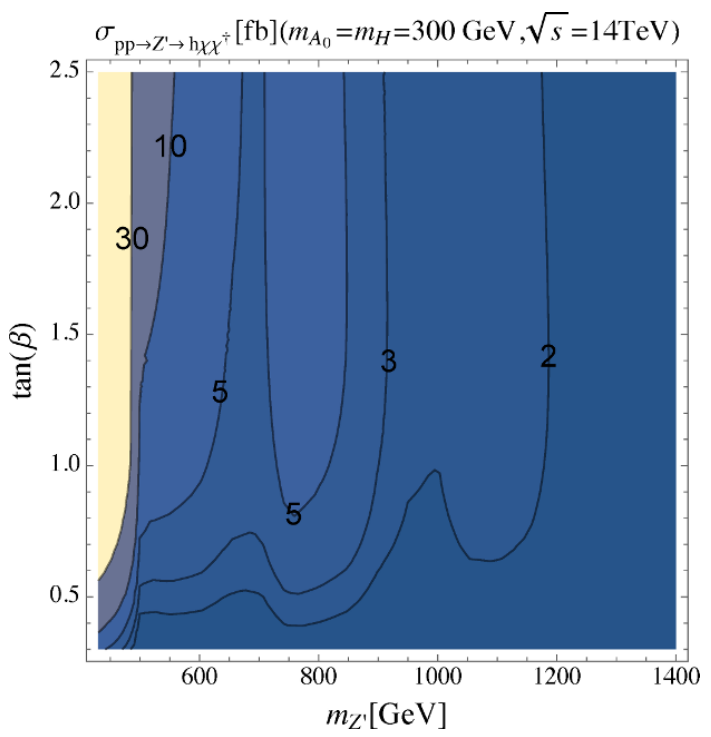

(c)

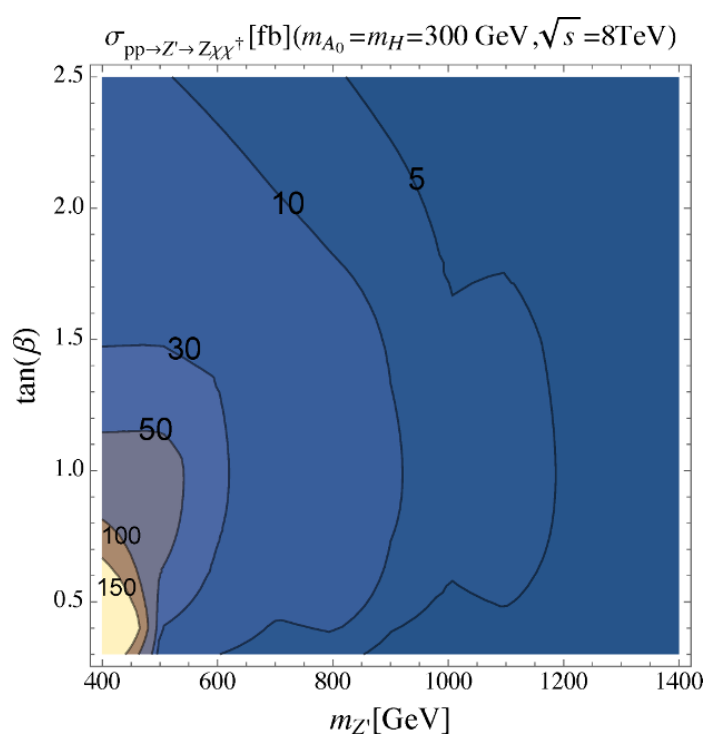

(b)

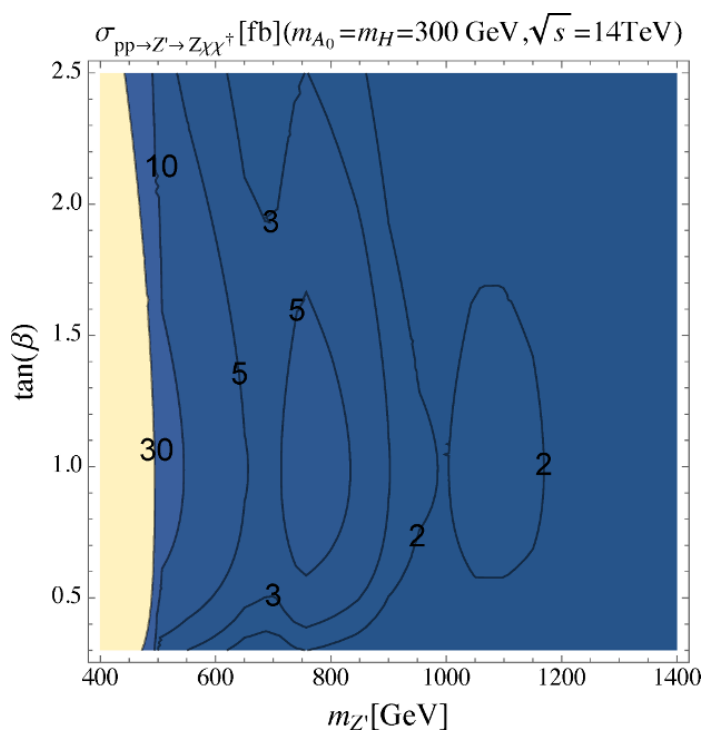

(d)

Figure 5. 2HDM model: cross-sections for the $Z^{\prime}$ mediated production of $h+\mathbb{E}_{T}(\mathrm{a}, \mathrm{c})$ and $Z+\mathbb{E}_{T}$ (b,d) at $\sqrt{s}=8 \mathrm{TeV}$ (top) and $\sqrt{s}=14 \mathrm{TeV}$ (bottom). The left-hand figures include the contribution from $h A_{0}$ together with $h Z$. We assume a $100 \%$ branching ratio to invisible decay $A_{0} \rightarrow \chi \chi^{\dagger}$ in $(\mathrm{a}, \mathrm{c})$ or $H \rightarrow \chi \chi^{\dagger}$ in $(\mathrm{b}, \mathrm{d})$. 


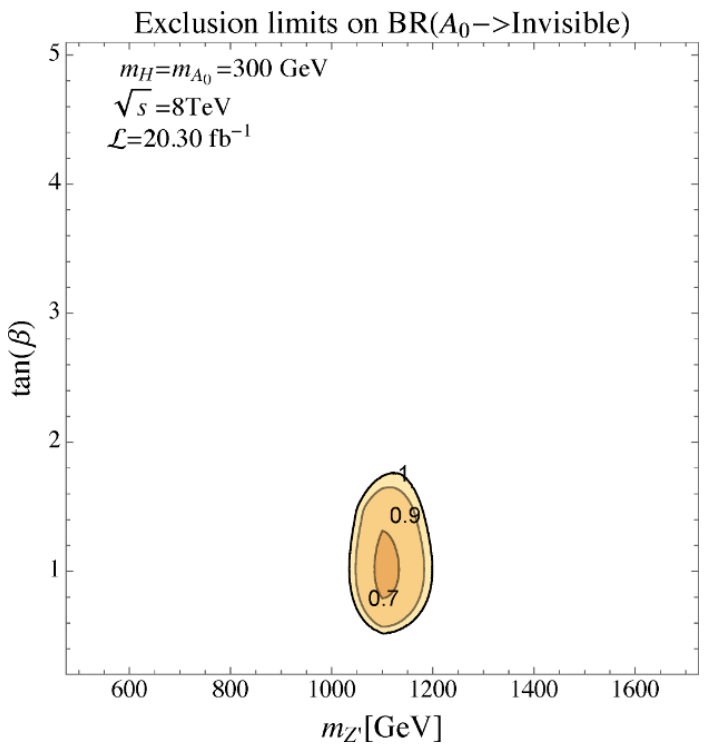

(a)

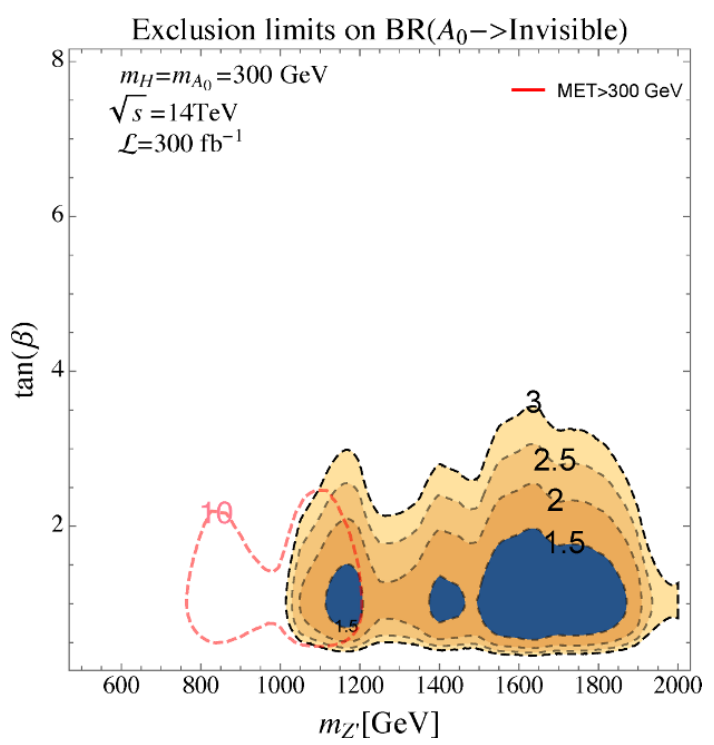

(c)

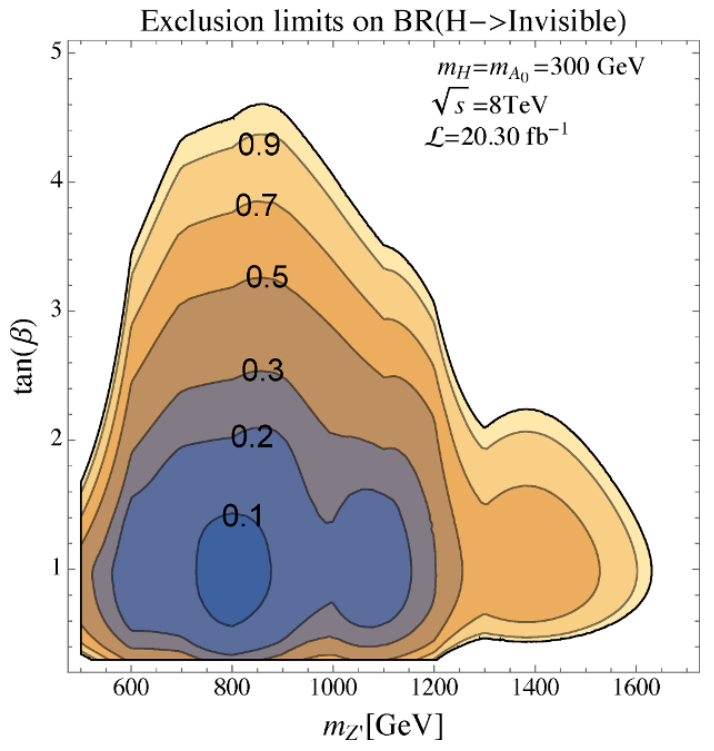

(b)

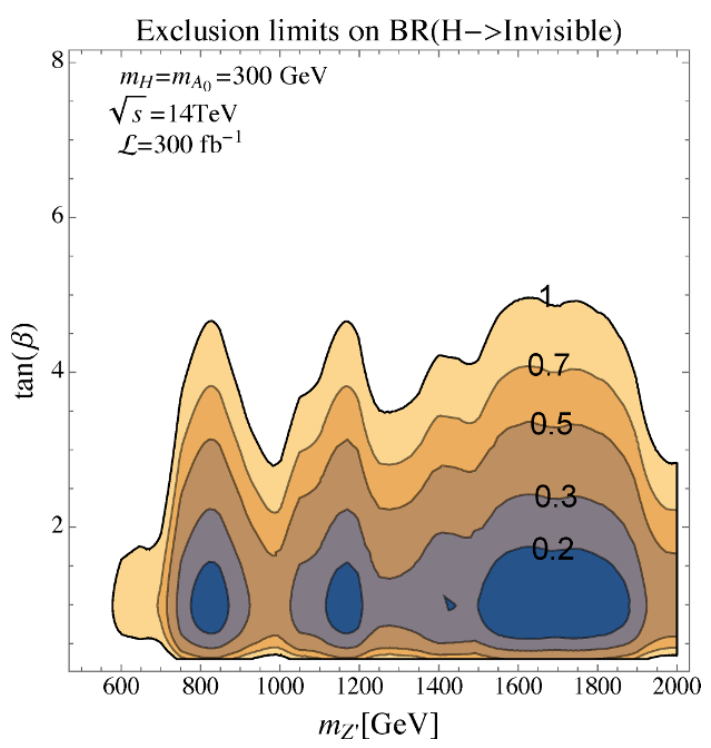

(d)

Figure 6. $2 \mathrm{HDM}$ model: limit on $\mathrm{BR}\left(A_{0} \rightarrow E_{T}\right)$ due to mono-Higgs analysis a) and on $\mathrm{BR}(H \rightarrow$ $E_{T}$ ) due to mono- $Z$ b) in the $M_{Z^{\prime}}-\tan \beta$ plane. c) and d) show projections at $14 \mathrm{TeV}$ with $300 \mathrm{fb}^{-1}$. The $Z^{\prime}$ production cross section has been set to saturate current and projected dijet resonance limits respectively, as explained in the text. Contour lines for upper limits greater than 1 in figure (c) are represented as dashed lines. The red curve in (c) represents the exclusion limit obtained from the less stringent cut $\mathbb{E}_{T} \geq 300 \mathrm{GeV}$ and closely mimics the limit obtained in [42] which, however, exploited a different analysis [70]. 
We take the decoupling limit $(\sin (\beta-\alpha)=1)$ so that the lighter CP-even Higgs is SM-like. Spontaneous symmetry breaking in the Higgs sector induces mixing between $Z^{\prime}$ and the SM $Z$ boson proportional to $\tan \beta$. The mixing is constrained by the precision electroweak measurement of the deviation of $\rho \equiv m_{W}^{2} / m_{Z}^{2} \cos \theta_{W}$ from unity [42]:

$$
\begin{aligned}
& \rho=1+\epsilon^{2}\left(\frac{m_{Z^{\prime}}^{2}-m_{Z}^{2}}{m_{Z}^{2}}\right), \\
& \epsilon \equiv \frac{\left(m_{Z}^{0}\right)^{2}}{m_{Z^{\prime}}^{2}-m_{Z}^{2}} \frac{g_{q} \cos \theta_{W}}{g} \sin ^{2} \beta,
\end{aligned}
$$

where $m_{Z}^{0}$ is the SM $Z$ boson mass in the absence of mixing. Furthermore, as $u_{R}$ is charged under the new $\mathrm{U}(1)_{Z^{\prime}}$ gauge symmetry, di-jet resonance searches for the $Z^{\prime}$ performed at hadron colliders constrain the $Z^{\prime}$ coupling to the initial state quarks (see figure 17). We apply these constraints and take the coupling of the $Z^{\prime}$ to the initial state quarks $\left(g_{q q Z^{\prime}}\right)$ to saturate the combined constraints. The couplings of $Z^{\prime}$ to $h A^{0}$ and $Z H$, which lead to mono-Higgs and mono- $Z$ signals, arise from the covariant derivative of the kinetic term of $H_{u}$.

We show in figure 5 the dependence of mono-Higgs and mono- $Z$ production crosssections at 8 and $14 \mathrm{TeV}$ on the $Z^{\prime}$ mass and $\tan \beta$. Both channels have similar dependence on the parameter space because the $Z^{\prime} A^{0} h$ and $Z^{\prime} Z H$ couplings are both inversely proportional to $\tan \beta$, but mono- $Z$ covers a larger parameter space with the same production cross-section. In figures 6 a) and b), we vary the branching ratio of $A^{0}$ and $H$ to DM and show the mono-Higgs and mono- $Z$ constraints on the $Z^{\prime}$ mass-tan $\beta$ plane. The $14 \mathrm{TeV}$ projection, performed with the procedure described in appendix $\mathrm{B}$, is shown in figure $6 \mathrm{c}$ ) and $\mathrm{d}$ ). Here again, we find that the mono- $Z$ channel is able to constrain a larger parameter region compared to the corresponding mono-Higgs channel. Let us again note that whether DM couples to $A^{0}$ or $H$ largely depends on the UV completion in the dark sector. Hence, both mono-Higgs and mono- $Z$ searches are equally useful for constraining this type of simplified model.

\subsection{Squarks with mono- $Z$}

We now consider a scenario which, in SUSY notation, involves a singlino as DM and 8 squarks as mediators:

$$
\mathcal{L} \supset g_{\mathrm{DM}} \sum_{i=1,2}\left(\widetilde{Q}_{L}^{i} \bar{Q}_{L}^{i}+\tilde{u}_{R}^{i} \bar{u}_{R}^{i}+\tilde{d}_{R}^{i} \bar{d}_{R}^{i}\right) \chi+\text { mass terms }+ \text { h.c. }
$$

Let us note that for the case where the mixing between left and right-handed squarks is zero, the mono-Higgs production cross-section is highly suppressed by the negligibly small quark masses. Although it is possible to introduce $A$-terms that could enhance the monoHiggs signal, this would in general lead to severe tuning in the quark Yukawa couplings (see e.g. [35]). Hence, we opt to leave out this possibility in this work. This is essentially the simplified model proposed in ref. [45] and used by the ATLAS collaboration to present their mono- $Z$ searches at Run I [48]. We show in figure 7 the constraint on $g_{\mathrm{DM}}$ as a 

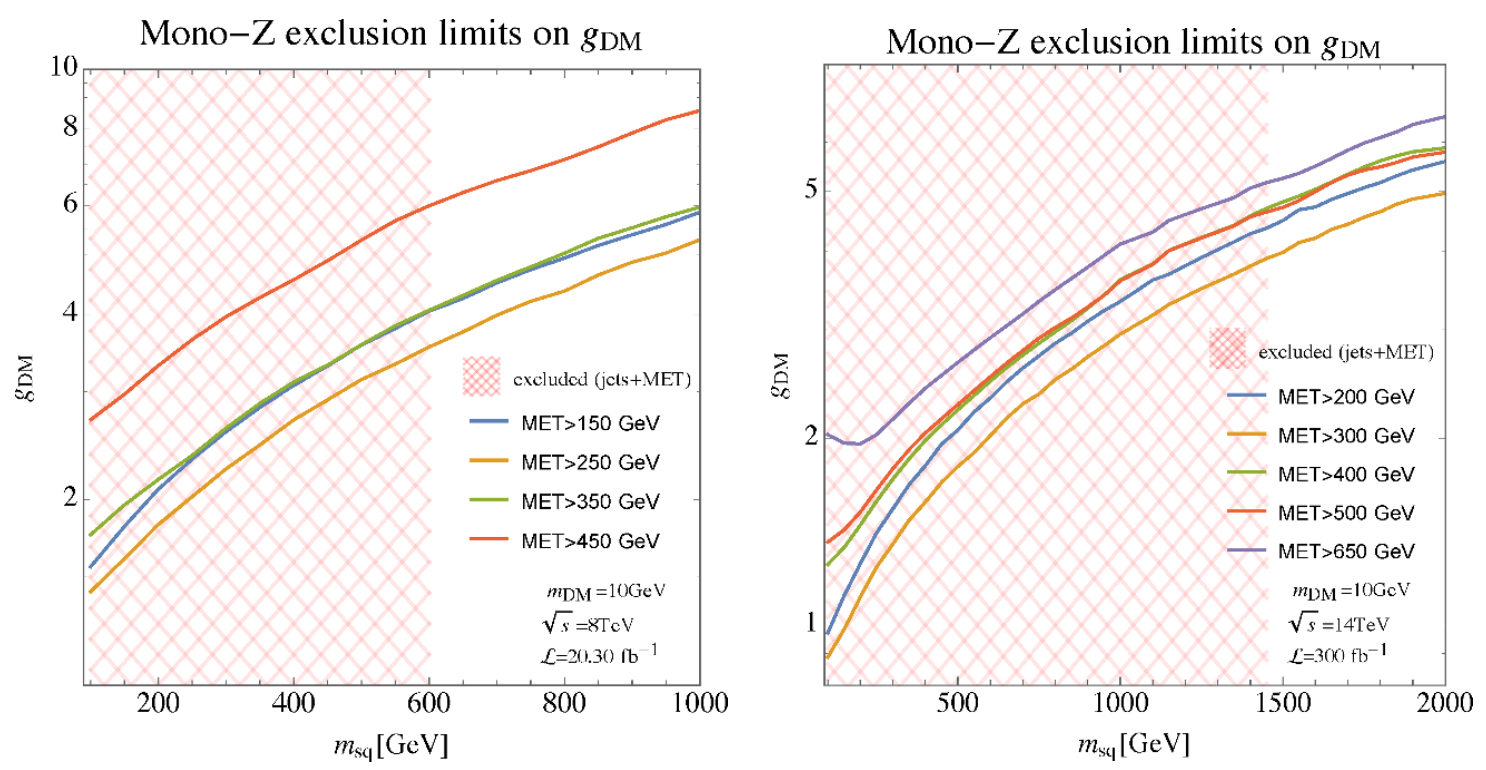

Figure 7. Squarks model: $95 \%$ exclusion limits on the quark-squark-dark matter coupling $g_{\mathrm{DM}}$. The mass of the dark matter is fixed to $m_{\mathrm{DM}}=10 \mathrm{GeV}$. The left panel shows the $8 \mathrm{TeV}$ constraints and the right panel shows the $14 \mathrm{TeV}$ projection with $300 \mathrm{fb}^{-1}$. The shaded region corresponds to values of the squark mass excluded by multi+jets $+\mathbb{E}_{T}$ analysis. Projections for jets $+\mathbb{E}_{T}$ limits are taken from ref. [71].

function of the mediating squark mass. We can see that in comparison to the mono-jets and jets $+\mathbb{E}_{T}$ constraints derived in [24], the constraints from mono- $Z$ production are very weak. $14 \mathrm{TeV}$ projections, performed with the procedure described in appendix B, are shown in the right panel of figure 7 . They improve the constraints, but are unlikely to be competitive with the di-jet and jets $+\mathbb{E}_{T}$ constraints.

Mono- $Z$ searches could in principle allow to access the compressed case, $m_{s q}-m_{\text {DM }} \ll$ $m_{s q}$, as shown in figure 8 . In this squeezed regime one can take advantage of the gluongluon initiated squark pair production, where the squarks then decay into dark matter plus soft jets. Attaching a $Z$ boson to one of the squark lines gives a process consistent with the mono- $Z$ cuts $^{5}$ and similar to the monojet topology. Even in this case, where the direct squark limits from the jets $+\mathbb{E}_{T}$ analysis [72] only places a constraint $m_{s q} \gtrsim 300 \mathrm{GeV}$, we find that mono- $Z$ searches are much weaker. The $14 \mathrm{TeV}$ projections shown in the right panel improve the limit and are indeed able to exclude compressed spectra up to $m_{s q} \gtrsim 100 \mathrm{GeV}$; nevertheless direct searches for squarks will continue to be much more powerful [71]. On the other hand, as explained in appendix B, our projections do not optimize the cuts to suppress the ratio of background over signal. Furthermore, we do not have access to the bin correlations: hence we conservatively assumed a $30 \%$ uncertainty in each bin. Future studies by the experimental collaborations are likely to improve the limits presented here, though it seems unlikely they will qualitatively change our conclusions.

\footnotetext{
${ }^{5}$ This is true only for compressed spectra: a larger mass separation would give rise to hard jet that would not pass the mono- $Z$ cuts on jet $p_{T}$.
} 

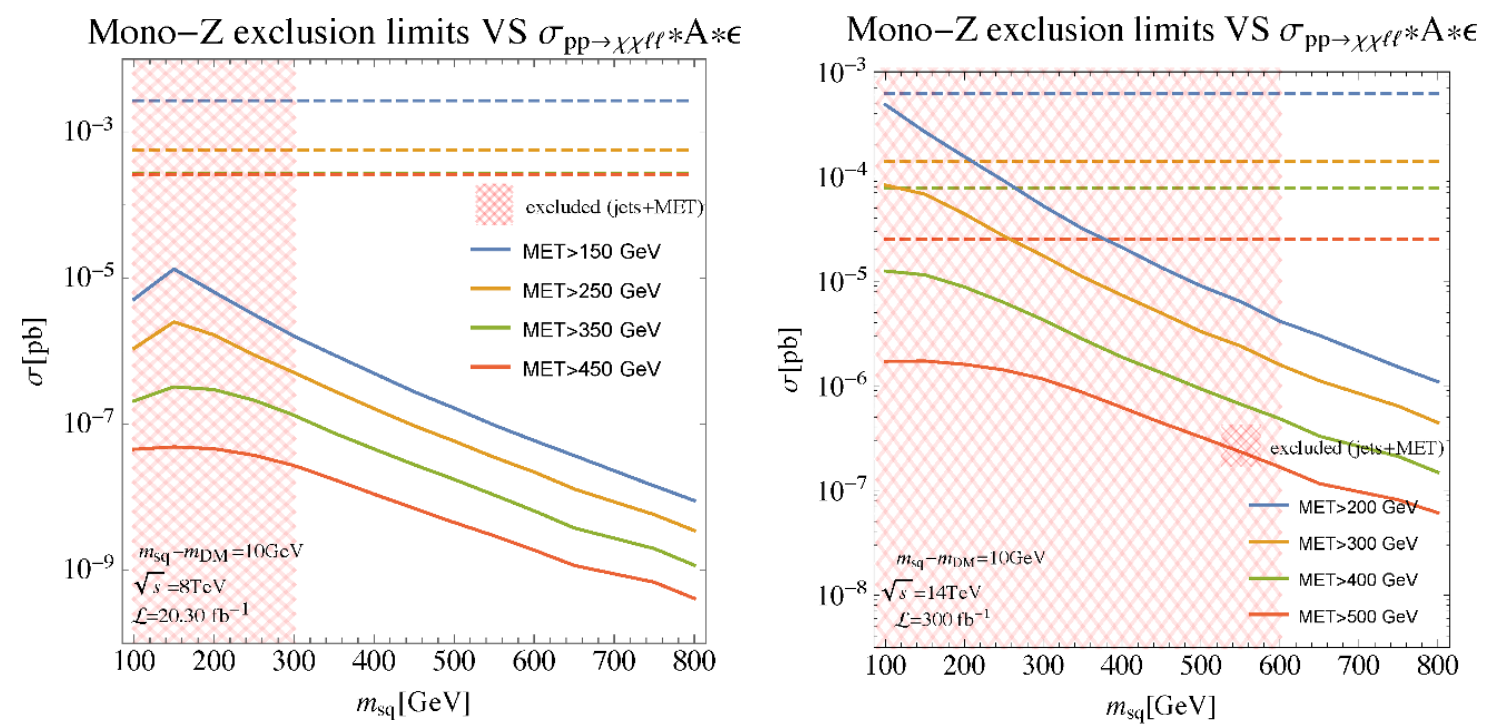

Figure 8. Squarks model: $95 \%$ exclusion limits on the mono- $Z$ cross-section, shown as dashed lines, and $95 \%$ exclusion limits on the cross-section after cuts for DM pair production in association with a $Z$, shown as solid lines. The mass of the dark matter is taken in the compressed region, $m_{s q}-m_{\mathrm{DM}}=10 \mathrm{GeV}$, since this enhances the cross-section (see text). The left panel shows the $8 \mathrm{TeV}$ constraints and the right panel shows the $14 \mathrm{TeV}$ projection with $300 \mathrm{fb}^{-1}$. Projections for jets $+\mathbb{E}_{T}$ limits are taken from ref. [71]. The four colors represent the four different $\mathbb{E}_{T}$ choices in the mono- $Z$ analysis.

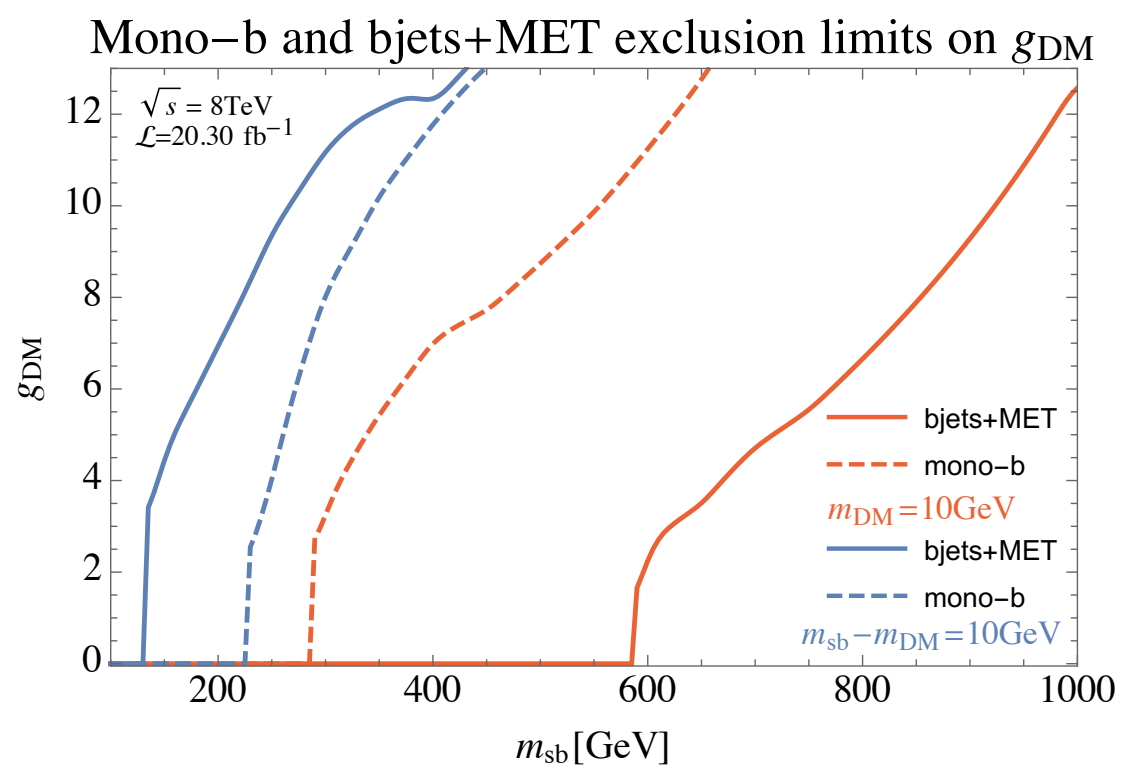

Figure 9. Sbottom model: $95 \%$ exclusion limits at $8 \mathrm{TeV}$ from mono-bjet and 2-bjet $+\mathbb{E}_{T}$ searches on the sbottom-bottom-DM coupling. The continuous red and blue lines represent bounds from $2 \mathrm{~b}+\mathbb{E}_{T}$ searches while dashed lines those from mono- $b$ searches. Different colors correspond to the limiting cases where the mass of the fermonic DM is taken light (red), $m_{\mathrm{DM}}=10 \mathrm{GeV}$, or in the compressed region (blue), $m_{\mathrm{sb}}-m_{\mathrm{DM}}=10 \mathrm{GeV}$. At the limit $g_{\mathrm{DM}} \rightarrow 0$ in the figure, it is implicitly assumed that $g_{\mathrm{DM}}$ is small enough such that sbottom pair productions are initiated solely by gluon-gluon processes, but $g_{\mathrm{DM}}$ is large enough that to make sbottoms decay promptly to DM. 


\subsection{Sbottoms with mono- $b$, mono- $h$ and mono- $Z$}

Similarly to the squark case, we take the Lagrangian as follows

$$
\mathcal{L}=g_{\mathrm{DM}}\left(\widetilde{Q}_{L}^{3} \bar{Q}_{L}^{3}+\tilde{b}_{R} \bar{b}_{R}^{i}\right) \chi+\text { mass terms }+g_{h}\left|H_{\mathrm{SM}}\right|^{2}\left(\left|\widetilde{Q}_{L}^{3}\right|^{2}+\left|\tilde{b}_{R}\right|^{2}\right)+\text { h.c. },
$$

where $H_{\mathrm{SM}}$ is the SM Higgs doublet. Notice that we do not normalize the sbottom coupling with the Higgs boson to the bottom Yukawa coupling. We consider first the direct sbottom search constraints in figure 9, comparing with the mono- $b$ search for the light DM $\left(m_{\mathrm{DM}}=\right.$ $10 \mathrm{GeV})$ and compressed region $\left(m_{\mathrm{sb}}-m_{\mathrm{DM}}=10 \mathrm{GeV}\right)$ cases. We can see that in the non-compressed region (i.e. for relatively large mass splitting between the sbottom and neutralino) the traditional sbottom searches dominate the constraints. On the other hand, in the compressed region, the mono- $b$ search becomes important. Note that in the noncompressed region, constraints lie around $m_{\mathrm{sb}}=600 \mathrm{GeV}$.

Next we compare these results to mono- $Z$ and mono- $h$ constraints in figure 10. Again we focus on two extremal cases: light DM and a compressed spectrum, where the process of gluon-gluon initiated sbottom pair production increases substantially the cross-section.

It is worth noting that different configurations translate into bounds on different combinations of couplings. For generic $m_{\mathrm{DM}}$, the mono- $Z$ search sets a limit on the sbottombottom-DM coupling, while mono- $h$ constrains the combination $g_{\mathrm{DM}} \sqrt{g_{h}}$. On the other hand, in the compressed regime the dependence on $g_{\mathrm{DM}}$ is lost. We show the projection at $14 \mathrm{TeV}$ in figure 11, performed with the procedure described in appendix B. Our results show that the mono- $Z$ analysis is never able to set a limit on perturbative values of the couplings. Stated in a different way, the cross-section rescaling needed to exclude a given point of the parameter space is nowhere close to one, both at LHC8 and LHC14, although the latter slightly improves over the former. This is not surprising, given the results of the previous subsection and the fact that the $Z$ boson does not distinguish between (s)quarks of different generations.

On the other hand, as shown in figure $10(\mathrm{c}, \mathrm{d})$ the mono- $h$ analysis is instead able to set a $\operatorname{limit}^{6}$ on the coupling $g_{h}$. The bound is further improved at LHC14, as shown in figure $11(\mathrm{c}, \mathrm{d})$.

\section{$2.5 s$-channel vector mediator}

Having investigated several models that can be constrained dominantly (at least in certain regions of parameter space) by various mono- $X$ searches, we now step back and consider models with an $s$-channel mediator that have been constrained previously by mono-jet, mono-Higgs and mono- $Z$.

We first consider the production of Higgs in association with a new massive gauge boson $Z^{\prime}$ which subsequently decays to DM. This mono-Higgs process occurs via an $s$ channel $Z^{\prime}$, and has been studied previously in ref. [41]. Our purpose here is to compare the constraints obtained there with di-jet and monojet constraints on the $Z^{\prime}$ mediator, which one expects to be important since the mediating $Z^{\prime}$ particle has interactions with

\footnotetext{
${ }^{6}$ The limit we found makes sense because of our normalization of $g_{h}$ in eq. (2.15).
} 


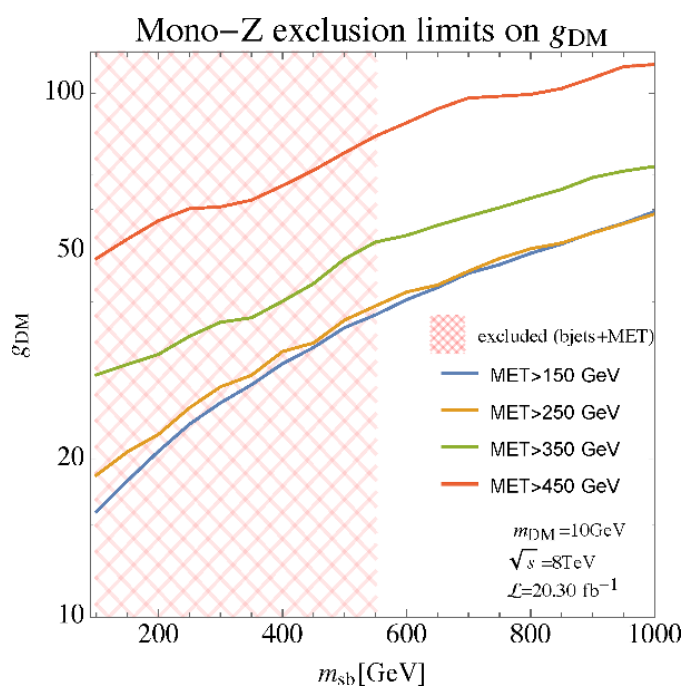

(a)

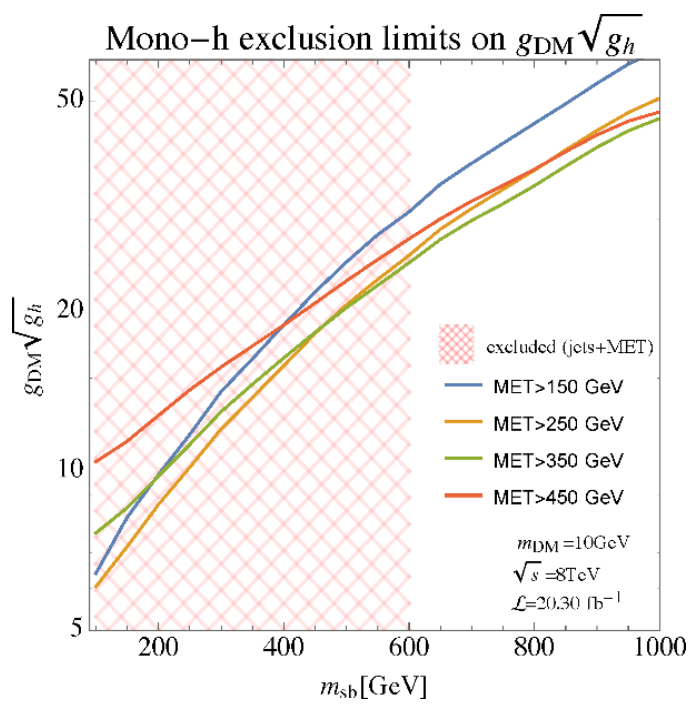

(c)

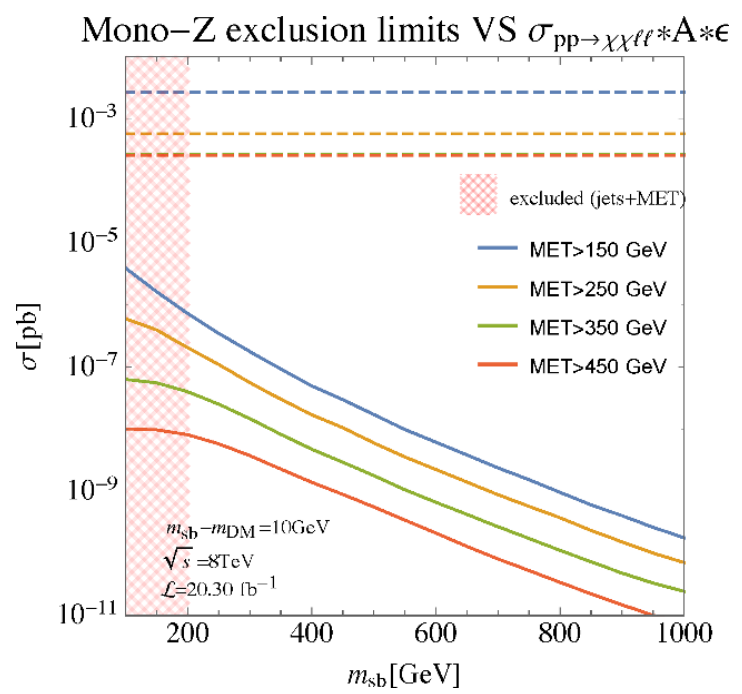

(b)

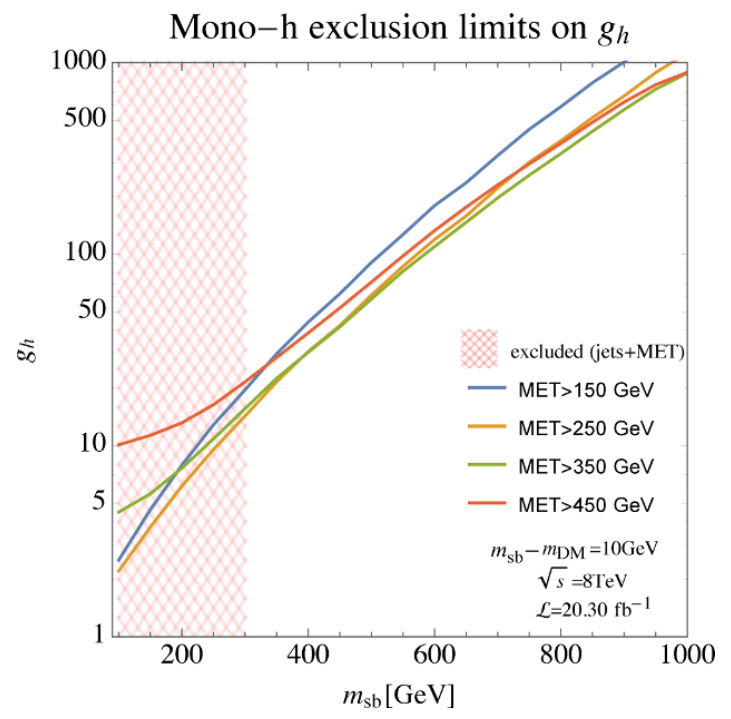

(d)

Figure 10. Sbottom model: $95 \%$ exclusion limits at $8 \mathrm{TeV}$ from various searches for the sbottom plus fermionic DM model. Different colors correspond to different choices of $\mathbb{E}_{T}$ cut in the respective analysis. a) Limits on the sbottom-bottom-DM coupling from a mono- $Z$ search as a function of the $\widetilde{b}$ mass. The mass of the dark matter is fixed to $m_{\mathrm{DM}}=10 \mathrm{GeV}$. b) $95 \%$ exclusion limits on the cross-section for the mono- $Z$ analysis, shown as dashed lines. $95 \%$ exclusion limits on the cross-section after cuts for DM in association with a $Z$ decaying into leptons, shown as solid lines. Here $m_{\mathrm{sb}}-m_{\mathrm{DM}}=10 \mathrm{GeV}$ and the DM is produced though a sbottom pair. c) Limits from a mono- $h$ search on the product $g_{\mathrm{DM}} \sqrt{g_{h}}$ as a function of the $\widetilde{b}$ mass, where $g_{h}$ is the Higgs-sbottom coupling. The mass of the dark matter is fixed to $m_{\mathrm{DM}}=10 \mathrm{GeV}$. d) Limits on $g_{h}$ from a mono- $h$ search in the compressed regime $m_{\mathrm{sb}}-m_{\mathrm{DM}}=10 \mathrm{GeV}$. 


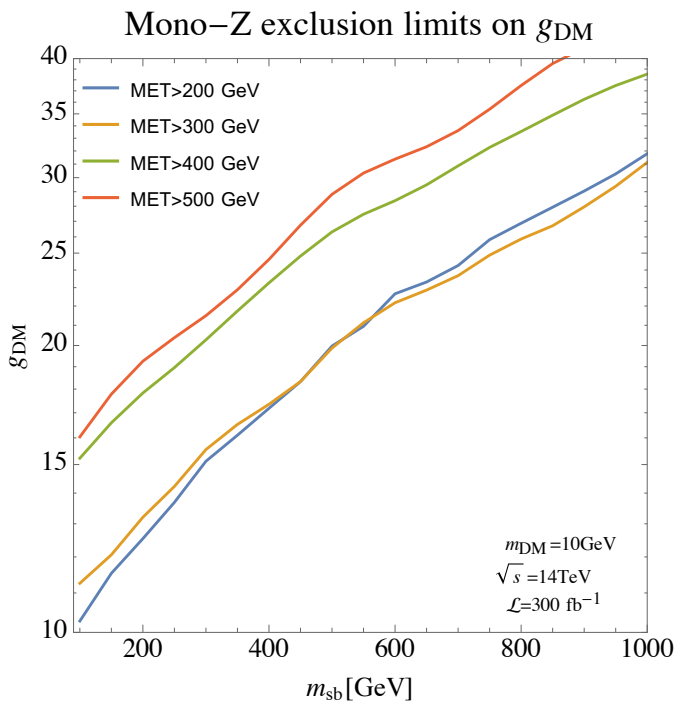

(a)

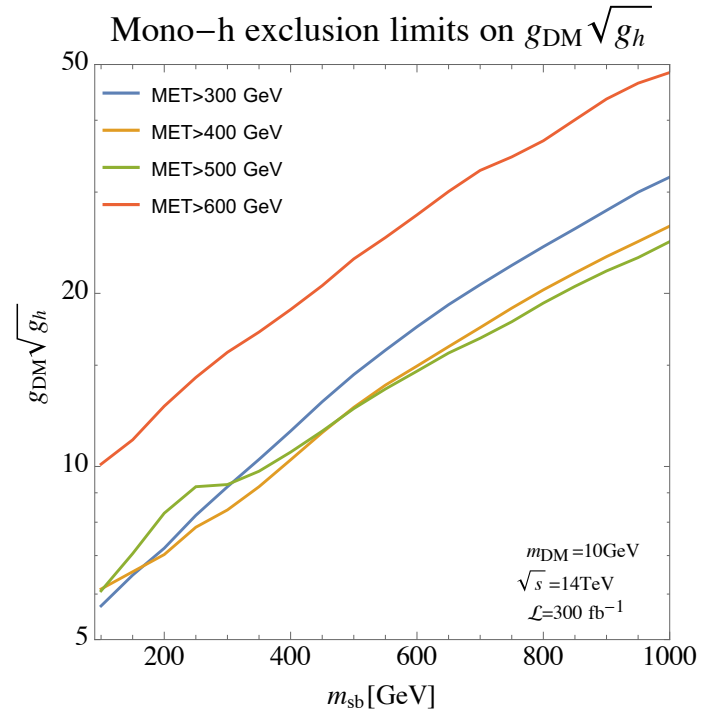

(c)

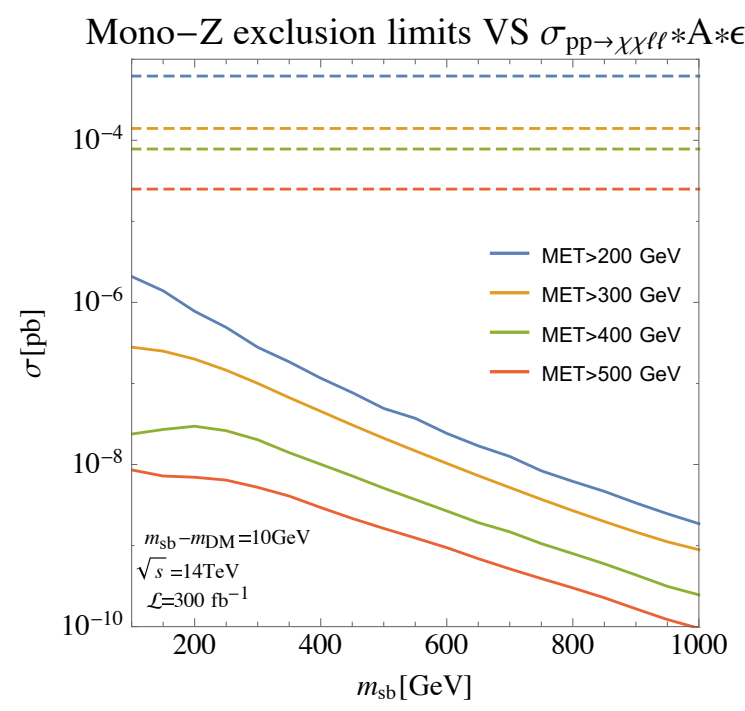

(b)

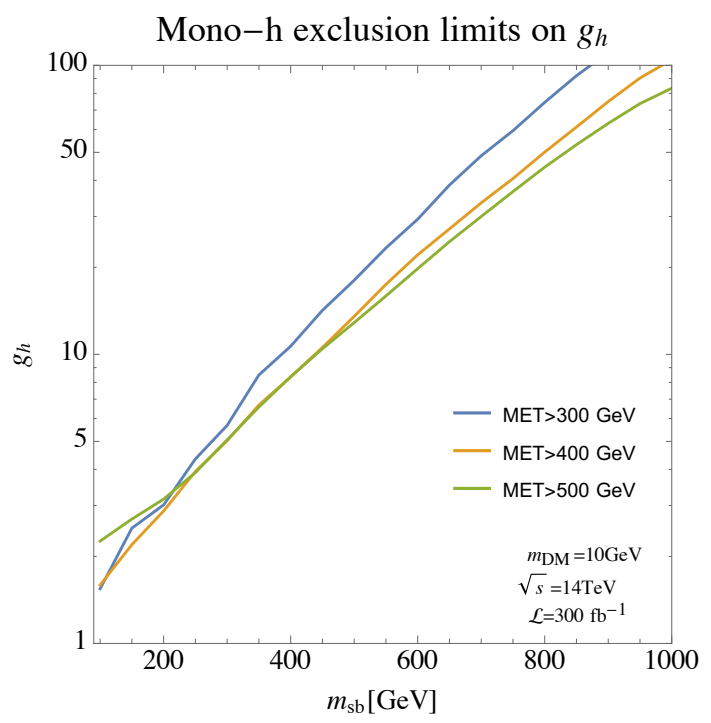

(d)

Figure 11. Sbottom model: projection at $14 \mathrm{TeV}$ with $300 \mathrm{fb}^{-1}$ of the $95 \%$ exclusion limits on the cross-section. Conventions are as in figure 10. We are not aware of existing mono- $b$ and $b$-jets $+\mathbb{E}_{T}$ projections at $14 \mathrm{TeV}$.

quarks as well as DM. We write the interaction Lagrangian of this simplified model as

$$
\begin{gathered}
\mathcal{L} \supset g_{q} Z_{\mu}^{\prime} \sum_{i=1,2}\left(\bar{Q}_{L}^{i} \gamma^{\mu} Q_{L}^{i}+\bar{u}_{R}^{i} \gamma^{\mu} u_{R}^{i}+\bar{d}_{R}^{i} \gamma^{\mu} d_{R}^{i}\right) \\
+g_{\mathrm{DM}} Z_{\mu}^{\prime} \bar{\chi} \gamma^{\mu} \chi+g_{H} m_{Z^{\prime}} h Z_{\mu}^{\prime} Z^{\prime \mu} .
\end{gathered}
$$

Such an interaction of a $Z^{\prime}$ with quarks and DM can originate from a baryon number gauge symmetry $\mathrm{U}(1)_{B}$, assuming $\mathrm{DM}$ is also gauged under $\mathrm{U}(1)_{B}$. We further assume that the $Z^{\prime}$ obtains its mass $m_{Z^{\prime}}$ from the spontaneous $\mathrm{U}(1)_{B}$ symmetry breaking due to a new 


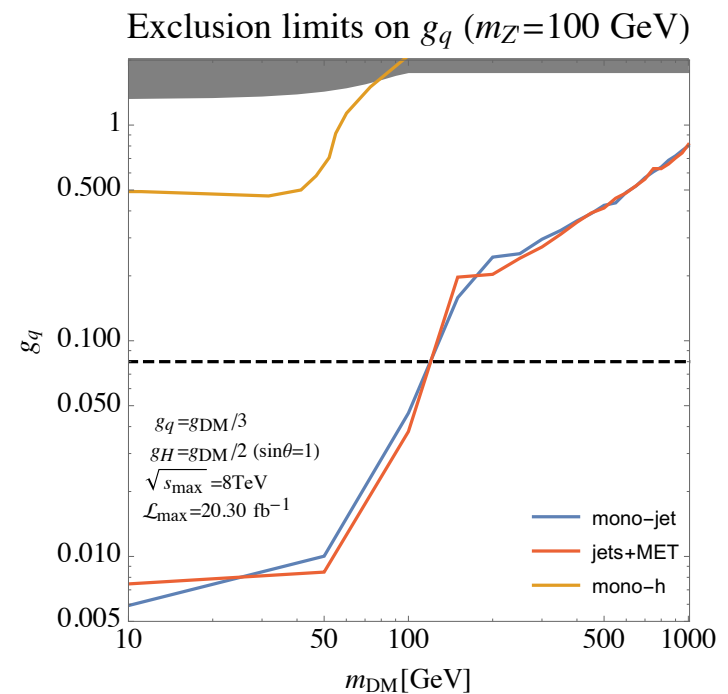

(a)

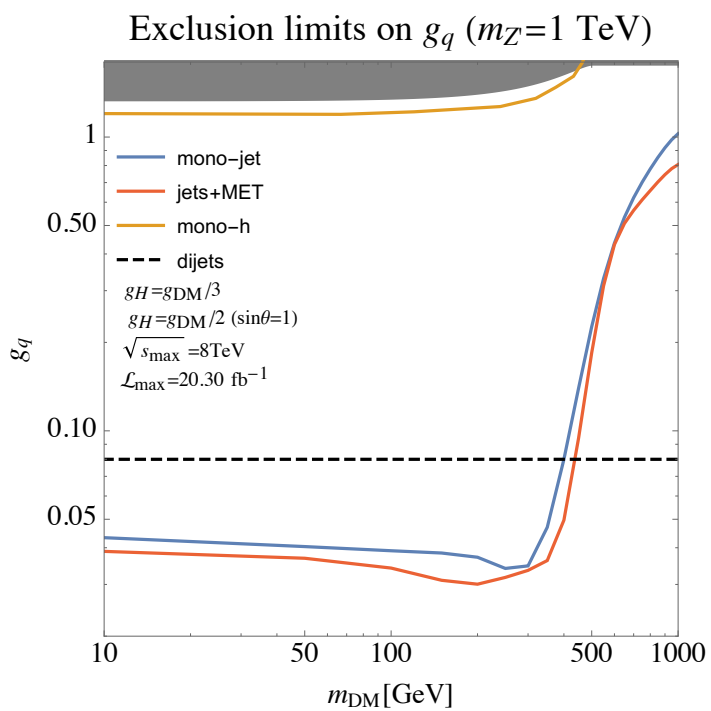

(b)

Figure 12. $s$-channel vector mediator model: comparison of exclusion limits at 95\% C.L. on the quark-Z' coupling $g_{q}$ as a function of the DM mass obtained from monojet, jets $+\mathbb{E}_{T}$, di-jet resonances and mono- $h$ searches. The latter is taken from ref. [41]. We show two benchmark points: $m_{Z^{\prime}}=100 \mathrm{GeV}$ and $m_{Z^{\prime}}=1000 \mathrm{GeV}, g_{q}=g_{\mathrm{DM}} / 3 . \sqrt{s_{\max }}$ and $\mathcal{L}_{\text {max }}$ represent the maximum energy and luminosity among the analyses used. A detailed list is reported in table 5 . The shaded region corresponds to the non-perturbative region defined by the condition on the $Z^{\prime}$ width $\Gamma_{Z^{\prime}}=m_{\mathrm{DM}}$.

scalar $h_{B}$ gauged under $\mathrm{U}(1)_{B}$. DM production associated with a Higgs is made possible by mixing the new scalar with the SM Higgs. $Z-Z^{\prime}$ mixing is not required to reproduce the mono- $h$ topology, and therefore the model is not constrained by precision electroweak measurements. See ref. [41] for a more detailed discussion. ${ }^{7}$ In this framework:

$$
g_{H}=\frac{m_{Z^{\prime}} \sin \theta}{v_{B}}, \quad \tan \theta=\frac{v_{B}}{v}, \quad\left\langle h_{B}\right\rangle=v_{B},
$$

where $v$ is the usual Higgs vev.

We compare constraints from mono-Higgs to those obtained from di-jet searches for the mediator, di-jet $+\mathbb{E}_{T}$, monojet, and mono-Higgs in figure 12. Different searches constrain different combinations of $g_{q}, g_{\mathrm{DM}}$ and $g_{H}$. To perform a meaningful comparison and make contact with the analysis already performed in ref. [41] we properly rescale one of their benchmarks and translate all the bounds to the quark and Higgs couplings:

$$
3 g_{q}=g_{\mathrm{DM}}=g_{B}, \quad g_{H}=3 g_{q} / 2
$$

where $g_{B}$ is the $Z^{\prime}$ gauge coupling, while the coupling to the Higgs boson $g_{H}$ has been taken at the formal limit of the perturbative regime consistent with eq. (2.17) in order to

\footnotetext{
${ }^{7}$ Another simplified model with the same DM production topology has been considered in ref. [41], where the $h Z^{\prime}$ production occurs via an $s$-channel SM $Z$ boson. In order to observe or constrain such a process at the LHC, however, one requires large $Z-Z^{\prime}$ mixing, which has already been disfavored by precision electroweak measurements. We do not consider this simplified model further.
} 
maximize the constraining power of mono-Higgs analysis. The only limits not present in the literature are those coming from jets $+\mathbb{E}_{T}$. These were obtained with a full recasting along the lines of ref. [24], using the minimal $Z^{\prime}$ width resulting from the couplings in eq. (2.16). Details are provided in appendix A. The limits from di-jets are taken directly from the literature $[73,74]$, taking into account the factor of $1 / 2$ difference in the normalization of the coupling.

For the choice of parameters in eq. (2.18), jets $+\mathbb{E}_{T}$ and monojet appear comparable and much more constraining than mono-Higgs searches. For heavier $Z^{\prime}$ masses (e.g. $M_{Z^{\prime}} \simeq$ $1.5 \mathrm{TeV})$, jets $+\mathbb{E}_{T}$ is less constraining, while the di-jet bound plays the dominant role.

\section{$2.6 s$-channel scalar mediator}

We next replace the vector $s$-channel mediator in the previous scenario with a scalar mediator in order to realize the DM production topology in the second row of table 1 . This is possible by introducing a singlet $S$ that acts as a portal between DM and the SM Higgs:

$$
\mathcal{L} \supset-y S \bar{\chi} \chi+\frac{1}{2} m_{h S}^{2} h S .
$$

Specifically, we consider the following Lagrangian:

$$
\mathcal{L}=\mathcal{L}_{\mathrm{SM}}+i \bar{\chi} \not \partial \chi+\frac{1}{2}\left(\partial_{\mu} S\right)^{2}-\frac{1}{2} m_{S}^{2} S^{2}-\eta\left(H_{\mathrm{SM}}^{\dagger} H_{\mathrm{SM}}\right) S-\lambda\left(H_{\mathrm{SM}}^{\dagger} H_{\mathrm{SM}}\right) S^{2}-y S \bar{\chi} \chi,
$$

where $H_{\mathrm{SM}}$ is the SM Higgs doublet. The SM Higgs sector is, as usual:

$$
\mathcal{L}_{\mathrm{SM}} \supset \frac{1}{2} m_{h}^{2}\left(H_{\mathrm{SM}}^{\dagger} H_{\mathrm{SM}}\right)-\frac{m_{h}^{2}}{2 v^{2}}\left(H_{\mathrm{SM}}^{\dagger} H_{\mathrm{SM}}\right)^{2}+\sum_{i}\left(y_{u}^{i} H_{\mathrm{SM}}^{\dagger} \bar{Q}_{L}^{i} u_{R}^{i}+y_{d}^{i} H_{\mathrm{SM}} \bar{Q}_{L}^{i} d_{R}^{i}\right) .
$$

This model was also considered in ref. [41], where they found that neither mono- $h$ nor mono$Z$ is strongly constraining. Here we consider whether a monojet search can be constraining on the parameter space of this model. We use the parameterization of a singlet mixed with the Higgs boson, defining

$$
\begin{aligned}
H_{\mathrm{SM}} & =\frac{1}{\sqrt{2}}\left(\begin{array}{c}
0 \\
v+h
\end{array}\right) \\
h & =\cos \theta h^{\prime}+\sin _{\theta} S^{\prime} \\
S & =-\sin _{\theta} h^{\prime}+\cos _{\theta} S^{\prime} \\
\tan 2 \theta & =\frac{2 \eta v}{m_{S}^{2}+\lambda v^{2}-m_{h}^{2}} .
\end{aligned}
$$

We obtain the Lagrangian in terms of the mass eigenstates $h^{\prime}$ and $S^{\prime}$. After the field redefinition, the new scalar $S^{\prime}$ couples to all quarks with strength $\frac{m_{q}}{v} \sin _{\theta}$. In addition, all the Higgs couplings will be rescaled by a factor of $\cos _{\theta}$. These shifts are taken into account in our analyses and plots.

We find that the constraints from the monojet search on such model are also generally very weak, as shown in figure 13. 


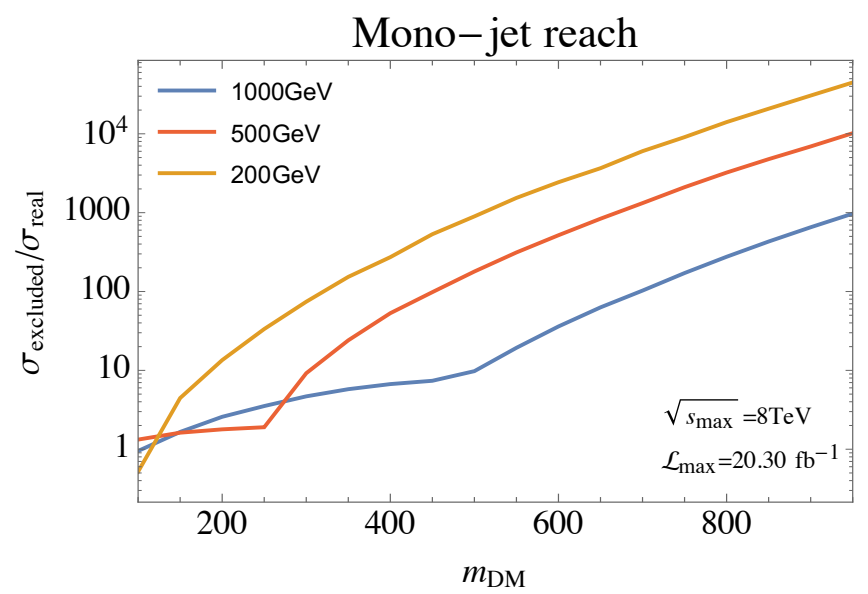

Figure 13. $s$-channel scalar mediator model: rescaling needed in the mono-jet $+\mathbb{E}_{T}$ cross-section in order for LHC8 to be sensitive. Different curves correspond to different values of the singlet mass $S$. $\sqrt{s_{\max }}$ and $\mathcal{L}_{\max }$ represent the maximum energy and luminosity amongst the analyses used. A detailed list is reported in table 5 .

\subsection{Inelastic squarks}

Until this point our simplified models have demanded that the dark matter not only be charge neutral, but also a singlet. In this (last) section we consider a scenario where the dark matter is not directly coupled to a squark-like particle, but is instead produced though an additional intermediate state. Although it is possible to build such a model using only singlet dark matter, one would engineer a rather complicated construction in order to produce sizable mono- $h$ and mono- $Z$ signals. ${ }^{8}$ Thus, for the sake of simplicity, here we abandon the singlet requirement in favor of a more elegant and simple model.

We study a model consisting of colored scalar mediators (the eight light flavor squarks) and two electroweak fermion doublets (Higgisnos, $\widetilde{H}_{1,2}$ ) acting as the mediators. The Higgsinos have a Dirac-like mass $\mu$-term, and their neutral components mix with a singlino $\chi(\mathrm{DM})$ via the SM Higgs to form mass eigenstates $\chi_{i}$, with $i=1-3$. Squarks couple to the singlino and $\widetilde{H}$ 's. The Lagrangian is:

$$
\begin{aligned}
\mathcal{L} \supset & -\frac{m_{S}}{2} \chi \chi-m_{D} \widetilde{H}_{1} \widetilde{H}_{2}-y_{1} \chi \widetilde{H}_{1} H_{\mathrm{SM}}-y_{2} \widetilde{\widetilde{H}}_{2} \bar{\chi} H_{\mathrm{SM}} \\
& +g_{\mathrm{DM}} \sum_{i=1,2}\left(\widetilde{Q}_{L}^{i} \bar{Q}_{L}^{i}+\tilde{q}_{R}^{i} \bar{q}_{R}^{i}\right) \chi+g_{\tilde{H}}\left({\widetilde{\widetilde{Q}_{L}}}_{L}^{i} q_{R}^{i} \widetilde{H}_{2}+\bar{q}_{R}^{i} \widetilde{Q}_{L}^{i} \widetilde{H}_{1}\right)+\text { h.c. }
\end{aligned}
$$

This model (and its pure electroweak subsector), being a generalization of a sector of the MSSM where the SUSY relations between gauge and Yukawa couplings have been relaxed, has been considered in the literature for many applications [19, 24, 36, 75, 76].

Here, we consider the production of $\chi \widetilde{H}$ through squarks in the $t$-channel at the LHC. $\widetilde{H}$ then decays into $Z(H)$ and $\chi$, giving a mono- $Z$ (mono- $h$ ) signature. In order for the

\footnotetext{
${ }^{8}$ Such a model would consist of a squark-like particle and two neutral states $\chi, \chi^{\prime}$. Sizable cross-section for mono- $h$ and mono- $Z$ are obtained through $Z-Z^{\prime}$ and $h-S$ mixing. Here $Z^{\prime}$ and $S$ are two additional vector and scalar fields.
} 
mono- $h$ and mono- $Z$ channels to compete with other direct searches we focus on the region of parameter space where the squarks predominantly decay to Higgsinos. In particular for our benchmark point we fix the decay branching ratios of the squarks to be $\operatorname{Br}(\tilde{q} \rightarrow$ $\left.q+\chi_{1}^{ \pm}\right): \operatorname{Br}\left(\tilde{q} \rightarrow q+\chi_{2,3}\right): \operatorname{Br}\left(\tilde{q} \rightarrow q+\chi_{1}\right) \simeq 6: 3: 1$ (this is achieved for example by choosing the ratio of the couplings $g_{\widetilde{H}} / g_{\mathrm{DM}}$ to be $\left.\sqrt{5}\right)$. Furthermore, we require that the neutrali Higgsinos to have equal branching rations for the decays into a $H$ or a $Z$ and the DM particle. While the full parameter space will not be explored in this paper, we identify three mass spectra as our benchmark scenarios, corresponding to non-compressed mass spectrum, compressed $\widetilde{H}-\chi_{1}$ mass spectrum, and compressed $\tilde{q}-\widetilde{H}$ mass spectrum.

For the scenario with non-compressed mass spectrum, the mass of $\chi_{2,3}$ and $\chi_{1}$ are $400 \mathrm{GeV}$ and $60 \mathrm{GeV}$ respectively. Note that the current LHC constraints on the electroweak production of electroweak-inos are irrelevant for this choice of parameters. In addition to $\chi \widetilde{H}$ production, the process $p p \rightarrow \chi_{2,3} \chi_{2,3} \rightarrow \chi_{1} \chi_{1} Z V(\equiv Z / h) \rightarrow \chi_{1} \chi_{1} \nu \bar{\nu} V$ also contributes to the mono- $Z(h)$ production. The reach of mono- $Z$ and mono- $h$ at $8 \mathrm{TeV}$ are shown in figure $14(\mathrm{a})$ on the $g_{\mathrm{DM}}-m_{s q}$ plane. On the same plot we show the constraints on $g_{\mathrm{DM}}$ from the $W Z+\mathbb{E}_{T}, W H+\mathbb{E}_{T}$, and the $W W+$ jets $+\mathbb{E}_{T}$ searches [66-68]. The first two constraints arise from the processes $p p \rightarrow \chi_{2,3} \chi_{1}^{ \pm} \rightarrow \chi_{1} \chi_{1} W^{ \pm} V$ via $t$-channel squarks, where $\chi_{1}^{ \pm}$is the charged Higgsino. The $W W+$ jets $+\mathbb{E}_{T}$ search corresponds to constraints from the direct squark decay to the $\mathrm{W}$ boson $\left(\tilde{q} \rightarrow j \chi_{1}^{ \pm} \rightarrow j \chi_{1} W^{ \pm}\right)$. This search tags the leptonic decay mode of the $W$ boson, and is more constraining than the standard jets $+\mathbb{E}_{T}$ searches. It can be observed that the mono- $Z / h$ search imposes weaker constraints than the $W W+$ jets $+\mathbb{E}_{T}$ search. Figure 14(b) shows LHC constraints $\left(W V+\mathbb{E}_{T}\right.$ searches) on the scenario with compressed $\widetilde{H}-\chi_{1}$ mass spectrum, with masses $\chi_{2,3}$ and $\chi_{1}$ set to $450 \mathrm{GeV}$ and $320 \mathrm{GeV}$ respectively. Overall the limits on $g_{\mathrm{DM}}$ are expectedly weakened in this compressed mass region. Even so, the $W V+\mathbb{E}_{T}$ searches are the more powerful probe of this parameter region compared to mono- $Z / h$. As the $W V+\mathbb{E}_{T}$ constraints alone are sufficient to overcome mono- $Z / h$, the $W W+$ jets $+\mathbb{E}_{T}$ constraint is not shown in the plot. While mono- $Z / h$ limits are expected to improve at $14 \mathrm{TeV}$ as shown in figure 14 (c) and figure $14(\mathrm{~d})$, the $8 \mathrm{TeV} W Z / W H / W W j+\mathbb{E}_{T}$ searches still outperform mono- $Z / h$.

Another scenario of interest lies in the compressed $\tilde{q}-\widetilde{H}$ mass region. We take squarks to be $10 \mathrm{GeV}$ heavier than $\widetilde{H}$, and vary the masses of $\widetilde{H}$ and $\chi$. Soft jets from the squark decay can escape detection, and the cascade decay of squarks contribute sizably to the $W V+\mathbb{E}_{T}$ channel. However, as can be observed in figure 15 , the $W Z+\mathbb{E}_{T}$ channel is more constraining than mono- $Z$, taking into account constraints from the QCD squark production and the process $p p \rightarrow \chi_{2,3} \chi_{1}^{ \pm} \rightarrow \chi \chi W^{ \pm} V\left(g_{\mathrm{DM}} \neq 0\right)$. In figure 16, one observes that the $W H+\mathbb{E}_{T}$ constraint from QCD squark production is dominant over mono- $h$ regardless of the values of $g_{\mathrm{DM}}$.

In summary, we do not find parameter space where mono- $Z / h$ is dominant over direct searches for the inelastic squark model.

\section{Conclusions}

It is essential to broadly explore DM simplified models at the LHC, elucidating how well the mono- $X$ and direct searches constrain each simplified model. In this paper, we pro- 
Mono-Z/h exclusion limits VS $g_{\mathrm{DM}}$

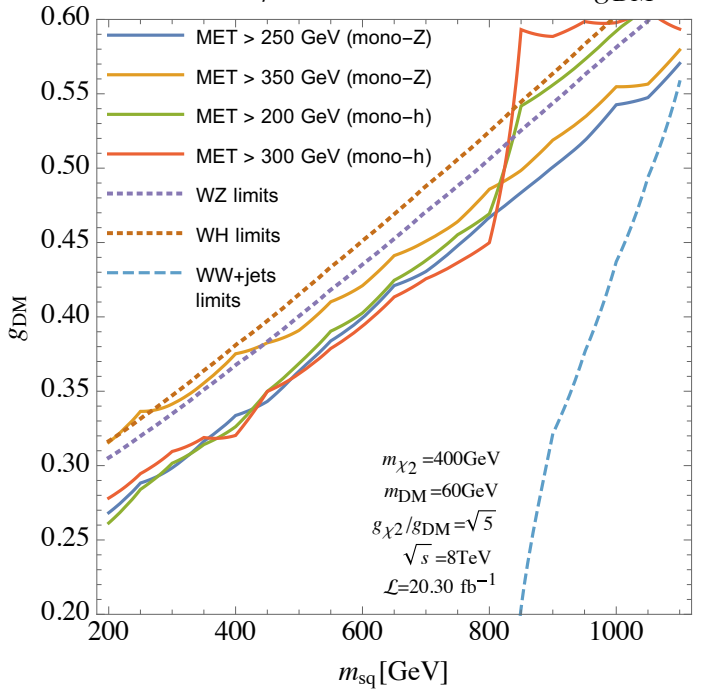

(a)

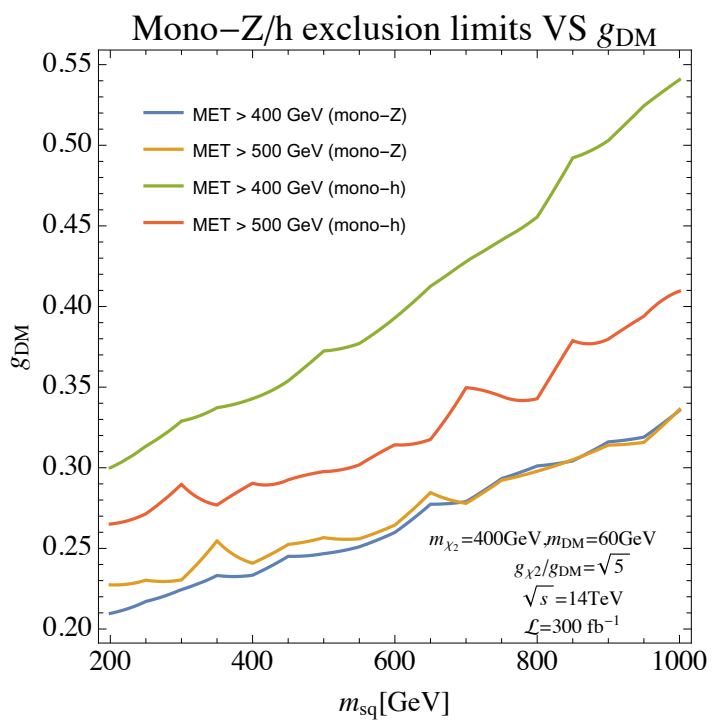

(c)

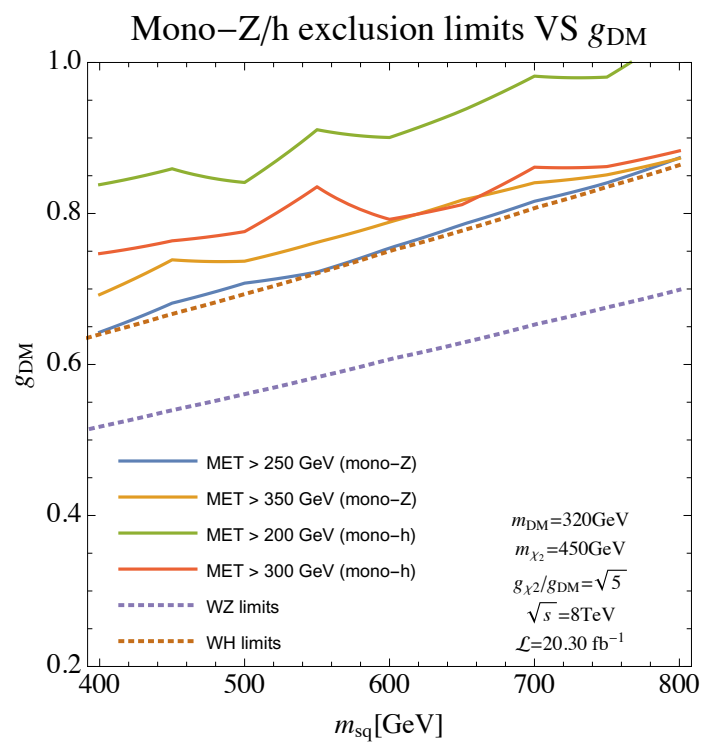

(b)

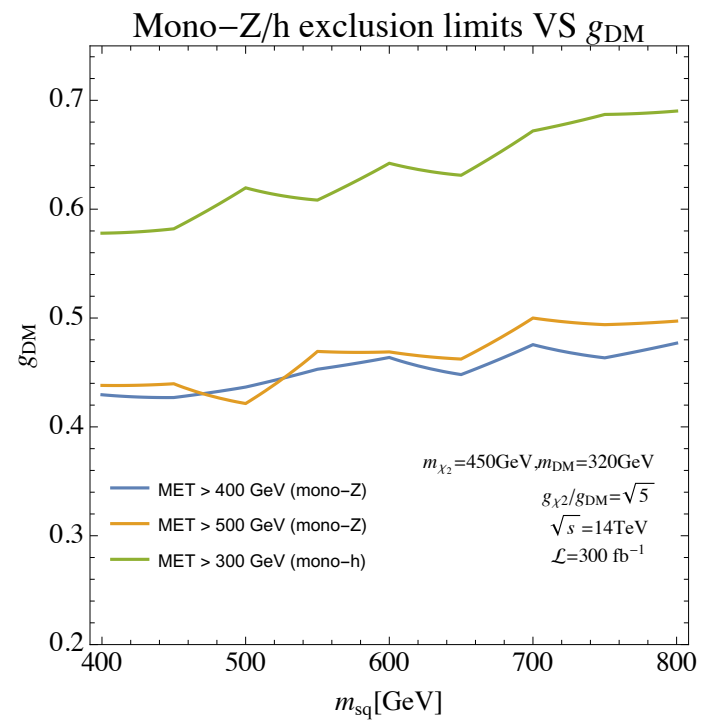

(d)

Figure 14. Inelastic squark model: mono- $Z$ and mono-Higgs, shown as solid lines with different colors corresponding to different $\mathbb{E}_{T}$ cuts, as well as $8 \mathrm{TeV} W Z, W W j$ and $W H+\mathbb{E}_{T}, 95 \%$ exclusion limits on the inelastic squark model, shown as dashed lines, with (a) DM mass $60 \mathrm{GeV}$, Higgsino mass $400 \mathrm{GeV}$, and (b) DM mass $320 \mathrm{GeV}$, Higgsino mass $450 \mathrm{GeV}$. The $W Z / W H / W W j+\mathbb{E}_{T}$ limits are estimated using efficiency tables and cross-section upper limits given in [66-68]. Panels (c) and (d) show the $14 \mathrm{TeV}$ projections at $300 \mathrm{fb}^{-1}$. 


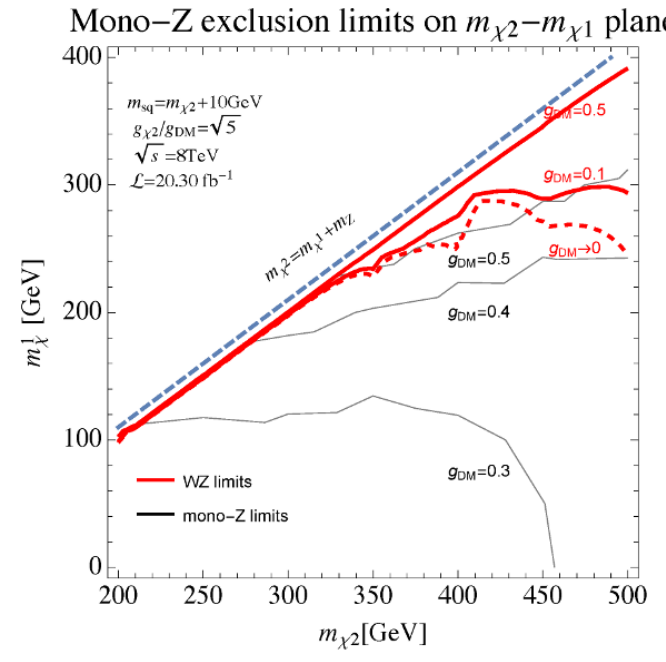

(a)

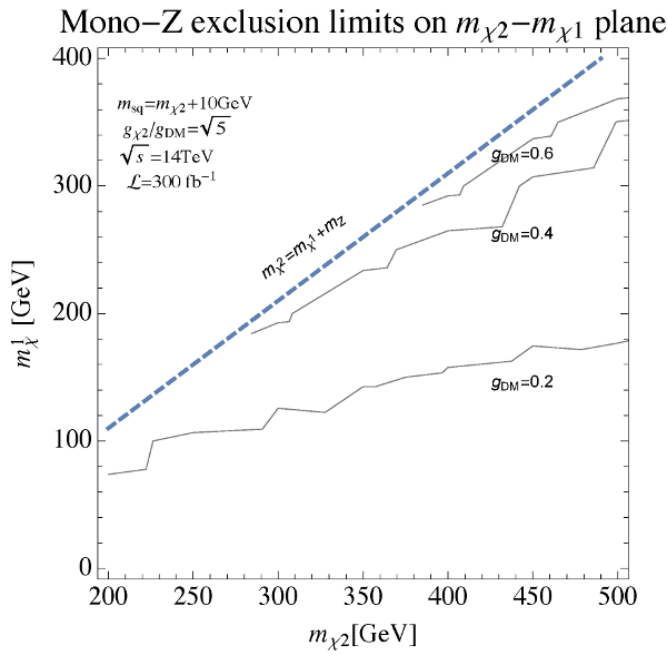

(b)

Figure 15. Inelastic squark model: mono- $Z$ limits (black lines) in the compressed mass region (squarks are $10 \mathrm{GeV}$ heavier than $\chi_{2}$ ) at $8 \mathrm{TeV}$ (a). The red dashed and solid lines represent limits from the electroweakino search in the $\mathrm{WZ}+\mathbb{E}_{T}$ final states [66]. The electroweakino search is dominant over mono- $Z$ in all parameter space investigated. The $14 \mathrm{TeV}$ projections are shown in panel (b).

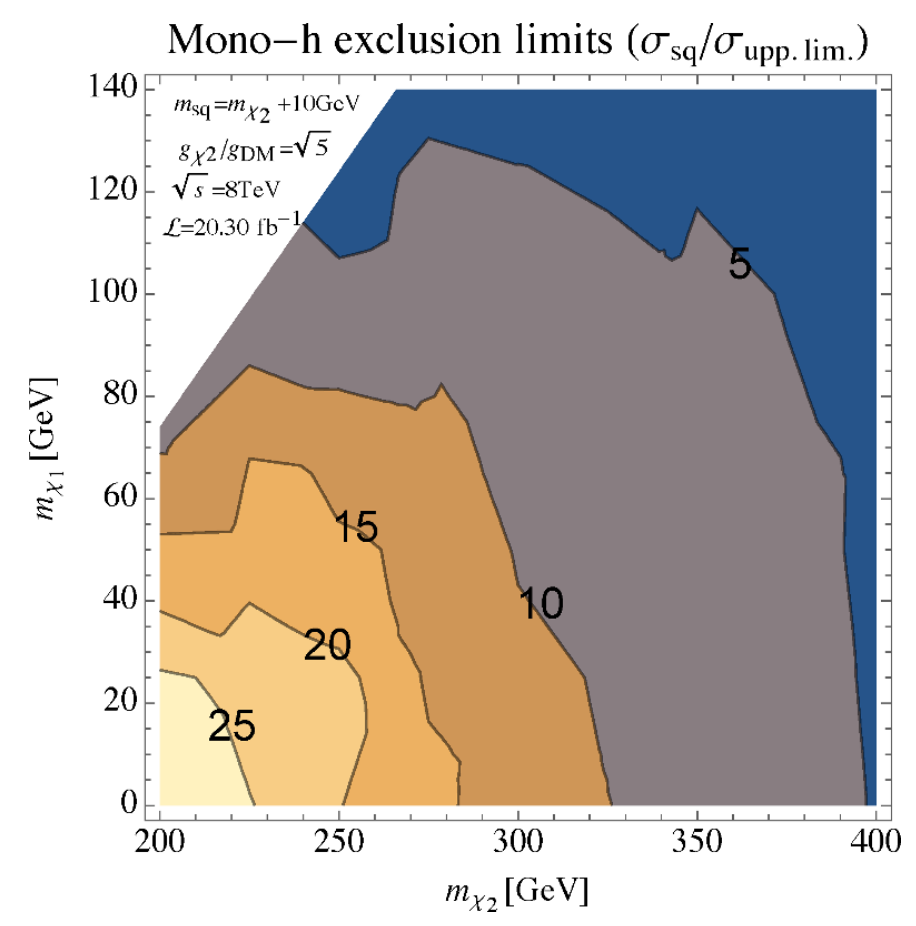

Figure 16. Inelastic squark model: mono- $h$ limits in the compressed mass region (squarks are $10 \mathrm{GeV}$ heavier than $\chi_{2}$ ). The contour lines represent the values of the squark production crosssection divided by the cross-section upper limits from the electroweakino search in the $\mathrm{WH}+\mathbb{E}_{T}$ final states [67]. The electroweakino search is dominant over mono- $h$ in all parameter space investigated. 
posed a set of simplified models covering mono- $X$ DM production topologies thoroughly, and we provided details of possible UV completions that realize the simplified model DM production topologies. Each model which produces a mono- $X$ signature through mediator decay to DM universally predicts other signatures, such as when the mediator decays back to the initial state particles that produced it (e.g. to a pair of jets). Generally, the direct search for the mediator through visible states such as di-jets and diboson will generate stronger constraints than the mono- $X$ constraints from DM decays, even when the DM coupling to the mediating particle is at the perturbative limit. However, each mono- $X$ search has a model, or region of parameter space, where the mono- $X$ signature dominates. This is summarized in table 3 .

While mono- $X$ signatures are not generic searches for DM, as they are typically not the dominant channel, they are a useful tool in the hunt for physics beyond the SM.

\section{Acknowledgments}

SPL is supported by JSPS Research Fellowships for Young Scientists and the Program for Leading Graduate Schools, MEXT, Japan. MP and KZ are supported by the DoE under contract DE-AC02-05CH11231. AV is supported by the Swiss National Science Foundation under grant no. PP00P2-163670.

\section{A Experimental analyses and simulation details}

In this appendix, we give descriptions of experimental analyses and simulation details of our study. For reference, we list all relevant collider searches utilized in our analysis in table 4 .

In the case of monojet (mono- $b$ ), (b-)jets $+\mathbb{E}_{T}$, and diboson signatures we made use of the cross-section limits on simplified models provided by experimental collaborations. ${ }^{9}$ For mono- $Z$ and mono- $h$ analyses we generate events and implement the cuts using the Madgraph [77], Pythia [78] and Delphes [79] pipeline. Our set of simplified models is implemented with the FeynRules package [80]. For all the other searches (mono-jet and jets $+\mathbb{E}_{T}$ ) we also performed a full simulation, following a somewhat different procedure: first we simulated events with MadGraph. The we showered using Pythia, which were then passed through Atom [81]. The procedure follows closely the one described in ref. [24] and we refer to it for all the details. All the simulated events used the minimal width resulting from the couplings of the simplified model.

Upper limits on mono- $X$ cross-sections are either taken from the experimental collaborations' reports, or extracted following the $C L_{S}$ prescription $[82,83]$. We summarize all LHC searches used in this work in table 5.

We report di-jet bounds on the $u_{R^{-}} Z^{\prime}$ coupling at $95 \%$ from three different sources $[42$, $73,84]$, which use different data sets and have somewhat different results, as shown in figure 17. The first (second) only provides bounds for $m_{Z^{\prime}} \geq 300 \mathrm{GeV}\left(m_{Z^{\prime}} \geq 150 \mathrm{GeV}\right)$. It should also be noted that in figure $17, Z^{\prime}$ presumably decays into jets with branching

\footnotetext{
${ }^{9}$ This method neglects finite width effects, as extensively discussed in [24].
} 


\begin{tabular}{|l|l|l|l|l|}
\hline Signature & Channel & Signal regions & search for & refs \\
\hline \multirow{3}{*}{ jet(s) $\left(+\mathbb{E}_{T}\right)$} & $2 j$ & di-jet resonance & $Z^{\prime}$ & {$[42,73,84]$} \\
& $2 j+\mathbb{E}_{T}$ & & $\tilde{q}$ & {$[72,85-89]$} \\
& $1 j+\mathbb{E}_{T}$ & & monojet & {$[64,90-93]$} \\
\hline \multirow{3}{*}{ b-jet(s) $\left(+\mathbb{E}_{T}\right)$} & $H(\rightarrow 2 b)+\mathbb{E}_{T}$ & $\mathbb{E}_{T}>150,200$, & mono- $h$ & {$[50]$} \\
& $2 b+\mathbb{E}_{T}$ & $300,400 \mathrm{GeV}$ & sbottom & {$[65]$} \\
& $1 b+\mathbb{E}_{T}$ & & mono- $b$ & {$[64]$} \\
\hline \multirow{3}{*}{ lepton(s) $\left(+j+\mathbb{E}_{T}\right)$} & $Z(\rightarrow l l) W(\rightarrow 2 j)+\mathbb{E}_{T}$ & $\mathbb{E}_{T}>150,250$, & $\chi_{1}^{ \pm} \chi_{2}^{0}$ & {$[66]$} \\
& $Z(\rightarrow l l)+\mathbb{E}_{T}$ & $350,450 \mathrm{GeV}$ & mono-Z & {$[48]$} \\
\hline combined & $W(\rightarrow l \nu) W(2 j)+j{ }^{\prime} \mathrm{s}+\mathbb{E}_{T}$ & & $\tilde{q}$ & {$[68]$} \\
\hline
\end{tabular}

Table 5. LHC searches used in this work.

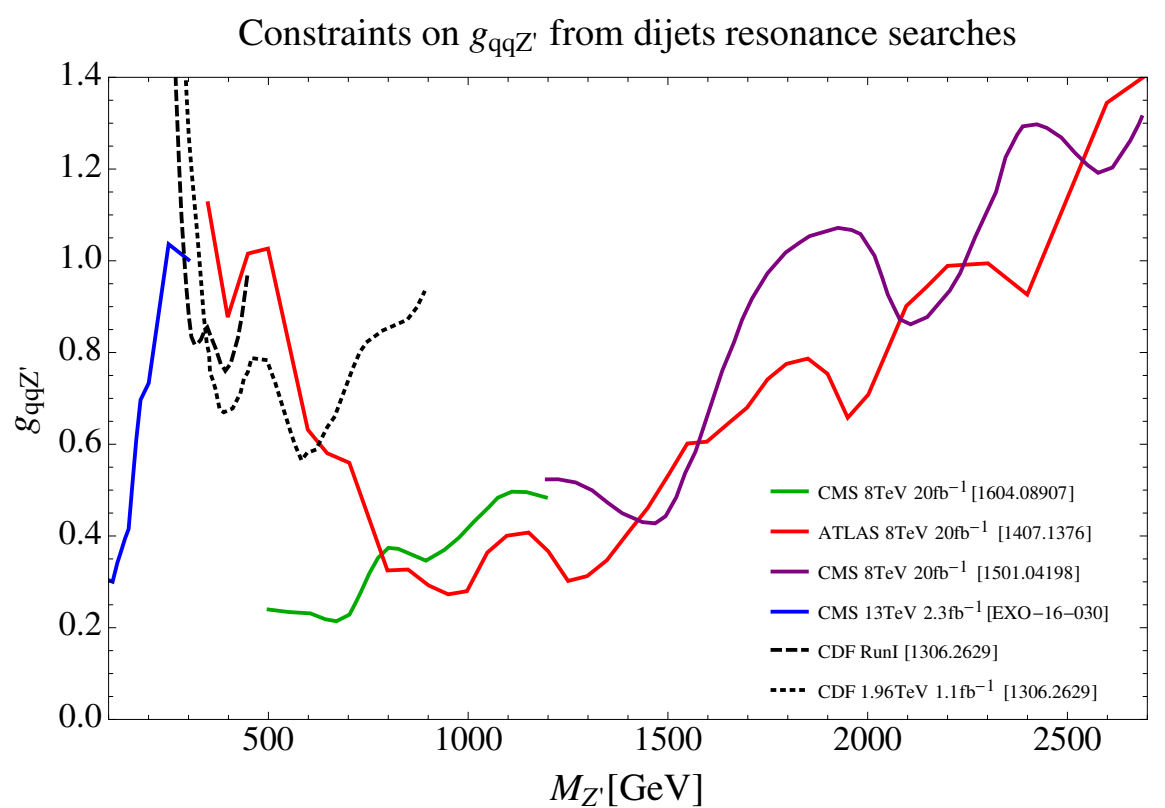

Figure 17. Upper limits on the $u_{R^{-}} Z^{\prime}$ coupling at $95 \%$ from di-jet resonance searches, taken from $[42,73,84]$, where different data sets are used to set the upper limits. For [73, 84], we have rescaled the coupling upper limits as presented in [94] by recalculating the di-jet production crosssection of the relevant processes to reflect the assumption in our model, which has $Z^{\prime}$ coupled only to $u_{R}$.

ratio $100 \%$. In our models, $Z^{\prime}$ can also decay into DM with a certain branching ratio, meaning that the $u_{R^{-}} Z^{\prime}$ coupling given in figure 17 has to be rescaled when $m_{Z^{\prime}}>2 m_{\mathrm{DM}}$. In our analysis, we calculate the partial width generated by the decay into DM and rescale the saturated di-jet constraints accordingly to take this into account.

\section{B $14 \mathrm{TeV}$ projections}

The $14 \mathrm{TeV}$ projected signal and background events are generated using the same pipeline. The total integrated luminosity is taken to be $300 \mathrm{fb}^{-1}$. The dominant SM background for 


\begin{tabular}{|c|c|c|c|c|c|c|c|c|}
\hline \multirow{3}{*}{\multicolumn{2}{|c|}{$8 \mathrm{TeV}($ mono- $Z$ ) }} & \multicolumn{2}{|c|}{$\mathbb{E}_{T}$ cut $[\mathrm{GeV}]$} & $>150$ & $>250$ & $>350$ & $>450$ & \\
\hline & & \multicolumn{2}{|c|}{ SM BG after cuts } & 52 & 7.2 & 1.4 & 0.4 & \\
\hline & & \multicolumn{2}{|c|}{ obs. limit [fb] } & 1.5 & 0.32 & 0.15 & 0.15 & \\
\hline \multirow{3}{*}{$14 \mathrm{TeV}$ (mono- $Z$ ) } & \multicolumn{2}{|c|}{$\mathbb{E}_{T}$ cut $[\mathrm{GeV}]$} & $>200$ & $>300$ & $>400$ & $>500$ & $>650$ & $>800$ \\
\hline & \multicolumn{2}{|c|}{ SM BG after cuts } & 311.9 & 66.7 & 33.4 & 6.2 & 1.0 & 0.2 \\
\hline & \multicolumn{2}{|c|}{ exp. limit [fb] } & 0.62 & 0.14 & 0.078 & 0.025 & 0.0099 & 0.0099 \\
\hline
\end{tabular}

Table 6. Signal regions, SM background events after applying cuts and cross-section times branching ratio upper limits at $95 \%$ C.L. for the mono- $Z$ search. The $8 \mathrm{TeV}$ results (background and observed cross-section times branching ratio upper limits) are taken from [48]. The expected crosssection times branching ratio upper limits for the $14 \mathrm{TeV}$ projections are estimated assuming a systematic uncertainty of $30 \%$. The total integrated luminosity is $300 \mathrm{fb}^{-1}$.

\begin{tabular}{|c|c|rrrr|}
\hline \multirow{3}{*}{$8 \mathrm{TeV}($ mono- $h)$} & $\notin_{T}$ cut $[\mathrm{GeV}]$ & $>150$ & $>200$ & $>300$ & $>400$ \\
\cline { 2 - 6 } & SM BG after cuts & 148 & 62 & 9.4 & 1.7 \\
\cline { 2 - 6 } & obs. limit $[\mathrm{fb}]$ & 3.7 & 1.3 & 0.45 & 0.20 \\
\hline \multirow{3}{*}{$14 \mathrm{TeV}$ (mono- $h)$} & $\mathbb{E}_{T}$ cut $[\mathrm{GeV}]$ & $>300$ & $>400$ & $>500$ & $>600$ \\
\cline { 2 - 6 } & SM BG after cuts & 402.9 & 79.4 & 19.4 & 7.6 \\
\cline { 2 - 6 } & exp. limit $[\mathrm{fb}]$ & 0.80 & 0.17 & 0.048 & 0.027 \\
\hline
\end{tabular}

Table 7. Signal regions, SM background events and cross-section times branching ratio upper limits at $95 \%$ C.L. for the mono- $h$ search. The $8 \mathrm{TeV}$ results (background and observed cross-section times branching ratio upper limits) are taken from [50]. The expected cross-section times branching ratio upper limits for the $14 \mathrm{TeV}$ projections are estimated assuming a systematic uncertainty of $30 \%$. The total integrated luminosity is $300 \mathrm{fb}^{-1}$.

mono- $Z$ is the diboson process $p p \rightarrow Z Z \rightarrow l^{+} l^{-} \nu \bar{\nu}$. In order to project the mono- $Z$ reach at $14 \mathrm{TeV}$, we tweak the $8 \mathrm{TeV}$ event selection criterion by increasing the $\mathbb{E}_{T}$ thresholds $(200,300,400,500,650$ and $800 \mathrm{GeV})$ to maintain approximately the same number of background events for the leading SM background contribution. Other event selection criteria are kept to be the same as in the $8 \mathrm{TeV}$ analysis. For mono- $h$, the $Z+$ jets, $t \bar{t}$ and diboson backgrounds are found to be important. Four SRs are defined according to the $\mathbb{E}_{T}$ thresholds at $14 \mathrm{TeV}: 300,400,500$ and $600 \mathrm{GeV}$ respectively. Similar to the mono- $Z$ projections, other event selection criteria are kept to be consistent with the $8 \mathrm{TeV}$ analysis. This prescription was validated by repeating it at $13 \mathrm{TeV}$ and comparing it with corresponding 2016 Run II analyses when these were available and found to yield good agreement.

The expected cross-section times branching ratio upper limit for each signal region is calculated using the $C L_{S}$ prescription. A systematic uncertainty of $30 \%$ is assumed in our estimate. In tables 6 and 7 we summarize the current status and prospects of mono- $Z$ and mono- $h$ searches. 
Open Access. This article is distributed under the terms of the Creative Commons Attribution License (CC-BY 4.0), which permits any use, distribution and reproduction in any medium, provided the original author(s) and source are credited.

\section{References}

[1] O. Nachtmann, A. Reiter and M. Wirbel, Single jet and single photon production in proton-anti-proton collisions and $e^{+} e^{-}$annihilation in a supersymmetric model, Z. Phys. C 27 (1985) 577 [INSPIRE].

[2] D.A. Dicus, S. Nandi and J. Woodside, Collider signals of a superlight gravitino, Phys. Rev. D 41 (1990) 2347 [INSPIRE].

[3] A. Brignole, F. Feruglio, M.L. Mangano and F. Zwirner, Signals of a superlight gravitino at hadron colliders when the other superparticles are heavy, Nucl. Phys. B 526 (1998) 136 [Erratum ibid. B 582 (2000) 759] [hep-ph/9801329] [INSPIRE].

[4] M. Brhlik, SUSY dark matter: Direct searches versus collider experiments, in the proceedings of the Conference on Dark matter in astrophysics and particle physics (DARK 1998), July 20-25, Heidelberg, Germany (1998), hep-ph/9810279 [INSPIRE].

[5] A. Birkedal, K. Matchev and M. Perelstein, Dark matter at colliders: a model independent approach, Phys. Rev. D 70 (2004) 077701 [hep-ph/0403004] [INSPIRE].

[6] F.J. Petriello, S. Quackenbush and K.M. Zurek, The invisible $Z^{\prime}$ at the CERN LHC, Phys. Rev. D 77 (2008) 115020 [arXiv:0803.4005] [INSPIRE].

[7] Y. Gershtein, F. Petriello, S. Quackenbush and K.M. Zurek, Discovering hidden sectors with mono-photon $Z^{\prime}$ o searches, Phys. Rev. D 78 (2008) 095002 [arXiv:0809.2849] [INSPIRE].

[8] M. Beltrán, D. Hooper, E.W. Kolb, Z.A.C. Krusberg and T.M.P. Tait, Maverick dark matter at colliders, JHEP 09 (2010) 037 [arXiv: 1002.4137] [INSPIRE].

[9] J. Goodman, M. Ibe, A. Rajaraman, W. Shepherd, T.M.P. Tait and H.-B. Yu, Constraints on light majorana dark matter from colliders, Phys. Lett. B 695 (2011) 185 [arXiv: 1005.1286] [INSPIRE].

[10] J. Goodman, M. Ibe, A. Rajaraman, W. Shepherd, T.M.P. Tait and H.-B. Yu, Constraints on dark matter from colliders, Phys. Rev. D 82 (2010) 116010 [arXiv:1008.1783] [InSPIRE].

[11] Y. Bai, P.J. Fox and R. Harnik, The Tevatron at the frontier of dark matter direct detection, JHEP 12 (2010) 048 [arXiv: 1005.3797] [INSPIRE].

[12] P.J. Fox, R. Harnik, J. Kopp and Y. Tsai, LEP shines light on dark matter, Phys. Rev. D 84 (2011) 014028 [arXiv: 1103.0240] [INSPIRE].

[13] P.J. Fox, R. Harnik, J. Kopp and Y. Tsai, Missing energy signatures of dark matter at the LHC, Phys. Rev. D 85 (2012) 056011 [arXiv:1109.4398] [INSPIRE].

[14] Y. Bai and T.M.P. Tait, Searches with mono-leptons, Phys. Lett. B 723 (2013) 384 [arXiv: 1208.4361] [INSPIRE].

[15] A. Friedland, M.L. Graesser, I.M. Shoemaker and L. Vecchi, Probing nonstandard standard model backgrounds with LHC monojets, Phys. Lett. B 714 (2012) 267 [arXiv:1111.5331] [INSPIRE].

[16] I.M. Shoemaker and L. Vecchi, Unitarity and monojet bounds on models for DAMA, CoGeNT and CRESST-II, Phys. Rev. D 86 (2012) 015023 [arXiv:1112.5457] [INSPIRE]. 
[17] G. Busoni, A. De Simone, E. Morgante and A. Riotto, On the validity of the effective field theory for dark matter searches at the LHC, Phys. Lett. B 728 (2014) 412 [arXiv: 1307.2253] [INSPIRE].

[18] S. Chang, R. Edezhath, J. Hutchinson and M. Luty, Effective WIMPs, Phys. Rev. D 89 (2014) 015011 [arXiv:1307.8120] [INSPIRE].

[19] H. An, L.-T. Wang and H. Zhang, Dark matter with t-channel mediator: a simple step beyond contact interaction, Phys. Rev. D 89 (2014) 115014 [arXiv:1308.0592] [INSPIRE].

[20] Y. Bai and J. Berger, Fermion portal dark matter, JHEP 11 (2013) 171 [arXiv:1308. 0612] [INSPIRE].

[21] H. Dreiner, D. Schmeier and J. Tattersall, Contact interactions probe effective dark matter models at the LHC, Europhys. Lett. 102 (2013) 51001 [arXiv:1303.3348] [INSPIRE].

[22] A. DiFranzo, K.I. Nagao, A. Rajaraman and T.M.P. Tait, Simplified models for dark matter interacting with quarks, JHEP 11 (2013) 014 [Erratum ibid. 01 (2014) 162] [arXiv: 1308.2679] [INSPIRE].

[23] O. Buchmueller, M.J. Dolan, S.A. Malik and C. McCabe, Characterising dark matter searches at colliders and direct detection experiments: Vector mediators, JHEP 01 (2015) 037 [arXiv: 1407.8257] [INSPIRE].

[24] M. Papucci, A. Vichi and K.M. Zurek, Monojet versus the rest of the world I: t-channel models, JHEP 11 (2014) 024 [arXiv: 1402.2285] [INSPIRE].

[25] G. Busoni, A. De Simone, J. Gramling, E. Morgante and A. Riotto, On the validity of the effective field theory for dark matter searches at the LHC, part II: complete analysis for the s-channel, JCAP 06 (2014) 060 [arXiv:1402.1275] [INSPIRE].

[26] G. Busoni, A. De Simone, T. Jacques, E. Morgante and A. Riotto, On the validity of the effective field theory for dark matter searches at the LHC part III: analysis for the t-channel, JCAP 09 (2014) 022 [arXiv: 1405.3101] [INSPIRE].

[27] D. Racco, A. Wulzer and F. Zwirner, Robust collider limits on heavy-mediator dark matter, JHEP 05 (2015) 009 [arXiv: 1502.04701] [INSPIRE].

[28] D. Abercrombie et al., Dark matter benchmark models for early LHC Run-2 searches: report of the ATLAS/CMS dark matter forum, arXiv:1507.00966 [INSPIRE].

[29] J. Abdallah et al., Simplified models for dark matter and missing energy searches at the LHC, arXiv:1409.2893 [INSPIRE].

[30] S.A. Malik et al., Interplay and characterization of dark matter searches at colliders and in direct detection experiments, Phys. Dark Univ. 9-10 (2015) 51 [arXiv:1409.4075] [INSPIRE].

[31] H. An, X. Ji and L.-T. Wang, Light dark matter and $Z^{\prime}$ dark force at colliders, JHEP 07 (2012) 182 [arXiv:1202.2894] [INSPIRE].

[32] M.T. Frandsen, F. Kahlhoefer, A. Preston, S. Sarkar and K. Schmidt-Hoberg, LHC and Tevatron bounds on the dark matter direct detection cross-section for vector mediators, JHEP 07 (2012) 123 [arXiv: 1204.3839] [INSPIRE].

[33] A. Alves, S. Profumo and F.S. Queiroz, The dark $Z^{\prime}$ portal: direct, indirect and collider searches, JHEP 04 (2014) 063 [arXiv: 1312.5281] [INSPIRE].

[34] M.R. Buckley, D. Feld and D. Goncalves, Scalar simplified models for dark matter, Phys. Rev. D 91 (2015) 015017 [arXiv: 1410.6497] [INSPIRE]. 
[35] K. Hamaguchi, S.P. Liew, T. Moroi and Y. Yamamoto, Isospin-violating dark matter with colored mediators, JHEP 05 (2014) 086 [arXiv:1403.0324] [INSPIRE].

[36] M. Garny, A. Ibarra, S. Rydbeck and S. Vogl, Majorana dark matter with a coloured mediator: collider vs. direct and indirect searches, JHEP 06 (2014) 169 [arXiv:1403.4634] [INSPIRE].

[37] M. Chala, F. Kahlhoefer, M. McCullough, G. Nardini and K. Schmidt-Hoberg, Constraining dark sectors with monojets and dijets, JHEP 07 (2015) 089 [arXiv: 1503.05916] [INSPIRE].

[38] A. Alves, A. Berlin, S. Profumo and F.S. Queiroz, Dirac-fermionic dark matter in $\mathrm{U}(1)_{X}$ models, JHEP 10 (2015) 076 [arXiv:1506.06767] [INSPIRE].

[39] D. Goncalves, P.A.N. Machado and J.M. No, Simplified models for dark matter face their consistent completions, Phys. Rev. D 95 (2017) 055027 [arXiv: 1611.04593] [INSPIRE].

[40] A.A. Petrov and W. Shepherd, Searching for dark matter at LHC with Mono-Higgs production, Phys. Lett. B 730 (2014) 178 [arXiv:1311.1511] [INSPIRE].

[41] L. Carpenter, A. DiFranzo, M. Mulhearn, C. Shimmin, S. Tulin and D. Whiteson, Mono-Higgs-boson: a new collider probe of dark matter, Phys. Rev. D 89 (2014) 075017 [arXiv: 1312.2592] [INSPIRE].

[42] A. Berlin, T. Lin and L.-T. Wang, Mono-Higgs detection of dark matter at the LHC, JHEP 06 (2014) 078 [arXiv: 1402 .7074] [INSPIRE].

[43] K. Ghorbani and L. Khalkhali, Mono-Higgs signature in fermionic dark matter model, arXiv: 1608.04559 [INSPIRE].

[44] N.F. Bell, Y. Cai and R.K. Leane, Mono-W dark matter signals at the LHC: simplified model analysis, JCAP 01 (2016) 051 [arXiv: 1512.00476] [INSPIRE].

[45] N.F. Bell, J.B. Dent, A.J. Galea, T.D. Jacques, L.M. Krauss and T.J. Weiler, Searching for dark matter at the LHC with a mono-Z, Phys. Rev. D 86 (2012) 096011 [arXiv:1209.0231] [INSPIRE].

[46] T. Lin, E.W. Kolb and L.-T. Wang, Probing dark matter couplings to top and bottom quarks at the LHC, Phys. Rev. D 88 (2013) 063510 [arXiv:1303.6638] [INSPIRE].

[47] E. Izaguirre, G. Krnjaic and B. Shuve, The Galactic Center excess from the bottom up, Phys. Rev. D 90 (2014) 055002 [arXiv: 1404.2018] [INSPIRE].

[48] ATLAS collaboration, Search for dark matter in events with a $Z$ boson and missing transverse momentum in pp collisions at $\sqrt{s}=8 \mathrm{TeV}$ with the ATLAS detector, Phys. Rev. D 90 (2014) 012004 [arXiv: 1404.0051] [INSPIRE].

[49] ATLAS collaboration, Search for dark matter in events with a hadronically decaying $W$ or $Z$ boson and missing transverse momentum in pp collisions at $\sqrt{s}=8 \mathrm{TeV}$ with the ATLAS detector, Phys. Rev. Lett. 112 (2014) 041802 [arXiv:1309.4017] [INSPIRE].

[50] ATLAS collaboration, Search for dark matter produced in association with a Higgs boson decaying to two bottom quarks in pp collisions at $\sqrt{s}=8 \mathrm{TeV}$ with the ATLAS detector, Phys. Rev. D 93 (2016) 072007 [arXiv:1510.06218] [InSPIRE].

[51] ATLAS collaboration, Search for new particles in events with one lepton and missing transverse momentum in pp collisions at $\sqrt{s}=8 \mathrm{TeV}$ with the ATLAS detector, JHEP 09 (2014) 037 [arXiv:1407.7494] [INSPIRE]. 
[52] CMS collaboration, Search for physics beyond the standard model in final states with a lepton and missing transverse energy in proton-proton collisions at $\sqrt{s}=8 \mathrm{TeV}$, Phys. Rev. D 91 (2015) 092005 [arXiv: 1408.2745] [INSPIRE].

[53] CMS collaboration, Search for dark matter in proton-proton collisions at $8 \mathrm{TeV}$ with missing transverse momentum and vector boson tagged jets, JHEP 12 (2016) 083 [arXiv: 1607.05764] [INSPIRE].

[54] CMS collaboration, Search for dark matter and unparticles produced in association with a $Z$ boson in proton-proton collisions at $\sqrt{s}=8 \mathrm{TeV}$, Phys. Rev. D 93 (2016) 052011 [arXiv: 1511.09375] [INSPIRE].

[55] N. Lopez, L.M. Carpenter, R. Cotta, M. Frate, N. Zhou and D. Whiteson, Collider bounds on indirect dark matter searches: the WW final state, Phys. Rev. D 89 (2014) 115013 [arXiv: 1403.6734] [INSPIRE].

[56] S. Gori, S. Jung and L.-T. Wang, Cornering electroweakinos at the LHC, JHEP 10 (2013) 191 [arXiv: 1307.5952] [INSPIRE].

[57] G.F. Giudice, T. Han, K. Wang and L.-T. Wang, Nearly degenerate gauginos and dark matter at the LHC, Phys. Rev. D 81 (2010) 115011 [arXiv:1004.4902] [INSPIRE].

[58] J. Brooke et al., Vector boson fusion searches for dark matter at the LHC, Phys. Rev. D 93 (2016) 113013 [arXiv:1603.07739] [INSPIRE].

[59] J. Andrea, B. Fuks and F. Maltoni, Monotops at the LHC, Phys. Rev. D 84 (2011) 074025 [arXiv:1106.6199] [INSPIRE].

[60] J. Wang, C.S. Li, D.Y. Shao and H. Zhang, Search for the signal of monotop production at the early LHC, Phys. Rev. D 86 (2012) 034008 [arXiv:1109.5963] [INSPIRE].

[61] J.-L. Agram, J. Andrea, M. Buttignol, E. Conte and B. Fuks, Monotop phenomenology at the Large Hadron Collider, Phys. Rev. D 89 (2014) 014028 [arXiv: 1311.6478] [INSPIRE].

[62] I. Boucheneb, G. Cacciapaglia, A. Deandrea and B. Fuks, Revisiting monotop production at the LHC, JHEP 01 (2015) 017 [arXiv: 1407.7529] [INSPIRE].

[63] J. D'Hondt, A. Mariotti, K. Mawatari, S. Moortgat, P. Tziveloglou and G. Van Onsem, Signatures of top flavour-changing dark matter, JHEP 03 (2016) 060 [arXiv:1511.07463] [INSPIRE].

[64] ATLAS collaboration, Search for pair-produced third-generation squarks decaying via charm quarks or in compressed supersymmetric scenarios in pp collisions at $\sqrt{s}=8 \mathrm{TeV}$ with the ATLAS detector, Phys. Rev. D 90 (2014) 052008 [arXiv:1407.0608] [INSPIRE].

[65] ATLAS collaboration, Search for direct third-generation squark pair production in final states with missing transverse momentum and two b-jets in $\sqrt{s}=8 \mathrm{TeV}$ pp collisions with the ATLAS detector, JHEP 10 (2013) 189 [arXiv:1308.2631] [INSPIRE].

[66] ATLAS collaboration, Search for direct production of charginos, neutralinos and sleptons in final states with two leptons and missing transverse momentum in pp collisions at $\sqrt{s}=8 \mathrm{TeV}$ with the ATLAS detector, JHEP 05 (2014) 071 [arXiv:1403.5294] [INSPIRE].

[67] CMS collaboration, Searches for electroweak neutralino and chargino production in channels with Higgs, $Z$ and $W$ bosons in pp collisions at 8 TeV, Phys. Rev. D 90 (2014) 092007 [arXiv: 1409.3168] [INSPIRE]. 
[68] ATLAS collaboration, Search for squarks and gluinos in events with isolated leptons, jets and missing transverse momentum at $\sqrt{s}=8 \mathrm{TeV}$ with the ATLAS detector, JHEP $\mathbf{0 4}$ (2015) 116 [arXiv: 1501.03555] [INSPIRE].

[69] J. Preskill, Gauge anomalies in an effective field theory, Annals Phys. 210 (1991) 323 [INSPIRE].

[70] ATLAS collaboration, Search for the bb decay of the Standard Model Higgs boson in associated $W / Z H$ production with the ATLAS detector (2013).

[71] T. Cohen et al., SUSY simplified models at 14, 33 and 100 TeV proton colliders, JHEP 04 (2014) 117 [arXiv: 1311.6480] [INSPIRE].

[72] ATLAS collaboration, Search for squarks and gluinos with the ATLAS detector in final states with jets and missing transverse momentum using $\sqrt{s}=8$ TeV proton-proton collision data, JHEP 09 (2014) 176 [arXiv:1405.7875] [InSPIRE].

[73] CMS collaboration, Search for narrow resonances in dijet final states at $\sqrt{s}=8 \mathrm{TeV}$ with the novel CMS technique of data scouting, Phys. Rev. Lett. 117 (2016) 031802 [arXiv: 1604.08907] [INSPIRE].

[74] CMS collaboration, Search for light vector resonances decaying to quarks at $13 \mathrm{TeV}$, CMS-PAS-EXO-16-030 (2016).

[75] R. Mahbubani and L. Senatore, The minimal model for dark matter and unification, Phys. Rev. D 73 (2006) 043510 [hep-ph/0510064] [INSPIRE].

[76] T. Cohen, J. Kearney, A. Pierce and D. Tucker-Smith, Singlet-doublet dark matter, Phys. Rev. D 85 (2012) 075003 [arXiv:1109.2604] [INSPIRE].

[77] J. Alwall, M. Herquet, F. Maltoni, O. Mattelaer and T. Stelzer, MadGraph 5: going beyond, JHEP 06 (2011) 128 [arXiv:1106.0522] [INSPIRE].

[78] T. Sjöstrand, S. Mrenna and P.Z. Skands, A brief introduction to PYTHIA 8.1, Comput. Phys. Commun. 178 (2008) 852 [arXiv:0710.3820] [INSPIRE].

[79] DELPHES 3 collaboration, J. de Favereau et al., DELPHES 3, a modular framework for fast simulation of a generic collider experiment, JHEP 02 (2014) 057 [arXiv:1307.6346] [INSPIRE].

[80] A. Alloul, N.D. Christensen, C. Degrande, C. Duhr and B. Fuks, FeynRules 2.0 - A complete toolbox for tree-level phenomenology, Comput. Phys. Commun. 185 (2014) 2250 [arXiv:1310.1921] [INSPIRE].

[81] I.-W. Kim, M. Papucci, K. Sakurai and A. Weiler, Atom: Automated Tests Of Models, in preparation.

[82] G. Cowan, K. Cranmer, E. Gross and O. Vitells, Asymptotic formulae for likelihood-based tests of new physics, Eur. Phys. J. C 71 (2011) 1554 [Erratum ibid. C 73 (2013) 2501] [arXiv: 1007.1727] [INSPIRE].

[83] B. Mistlberger and F. Dulat, Limit setting procedures and theoretical uncertainties in Higgs boson searches, arXiv:1204.3851 [INSPIRE].

[84] ATLAS collaboration, Search for new phenomena in the dijet mass distribution using pp collision data at $\sqrt{s}=8$ TeV with the ATLAS detector, Phys. Rev. D 91 (2015) 052007 [arXiv: 1407.1376] [INSPIRE]. 
[85] CMS collaboration, Search for new physics in the multijet and missing transverse momentum final state in proton-proton collisions at $\sqrt{s}=8 \mathrm{TeV}$, JHEP 06 (2014) 055 [arXiv: 1402.4770] [INSPIRE].

[86] CMS collaboration, Search for supersymmetry in hadronic final states with missing transverse energy using the variables $\alpha_{T}$ and b-quark multiplicity in pp collisions at $\sqrt{s}=8$ TeV, Eur. Phys. J. C 73 (2013) 2568 [arXiv:1303.2985] [inSPIRE].

[87] ATLAS collaboration, Search for squarks and gluinos using final states with jets and missing transverse momentum with the ATLAS detector in $\sqrt{s}=7$ TeV proton-proton collisions, Phys. Lett. B 701 (2011) 186 [arXiv:1102.5290] [INSPIRE].

[88] CMS collaboration, Search for new physics in the multijet and missing transverse momentum final state in proton-proton collisions at $\sqrt{s}=7 \mathrm{TeV}$, Phys. Rev. Lett. 109 (2012) 171803 [arXiv: 1207.1898] [INSPIRE].

[89] CMS collaboration, Search for supersymmetry in final states with missing transverse energy and 0, 1, 2, or at least 3 b-quark jets in $7 \mathrm{TeV}$ pp collisions using the variable $\alpha_{T}$, JHEP 01 (2013) 077 [arXiv:1210.8115] [INSPIRE].

[90] ATLAS collaboration, Search for new phenomena in final states with an energetic jet and large missing transverse momentum in pp collisions at $\sqrt{s}=8 \mathrm{TeV}$ with the ATLAS detector, Eur. Phys. J. C 75 (2015) 299 [arXiv:1502.01518] [INSPIRE].

[91] CMS collaboration, Search for dark matter, extra dimensions and unparticles in monojet events in proton-proton collisions at $\sqrt{s}=8$ TeV, Eur. Phys. J. C 75 (2015) 235 [arXiv: 1408.3583] [INSPIRE].

[92] ATLAS collaboration, Search for dark matter candidates and large extra dimensions in events with a jet and missing transverse momentum with the ATLAS detector, JHEP 04 (2013) 075 [arXiv:1210.4491] [INSPIRE].

[93] CMS collaboration, Search for dark matter and large extra dimensions in monojet events in pp collisions at $\sqrt{s}=7 \mathrm{TeV}$, JHEP 09 (2012) 094 [arXiv:1206.5663] [INSPIRE].

[94] B.A. Dobrescu and F. Yu, Coupling-mass mapping of dijet peak searches, Phys. Rev. D 88 (2013) 035021 [arXiv: 1306.2629] [INSPIRE]. 